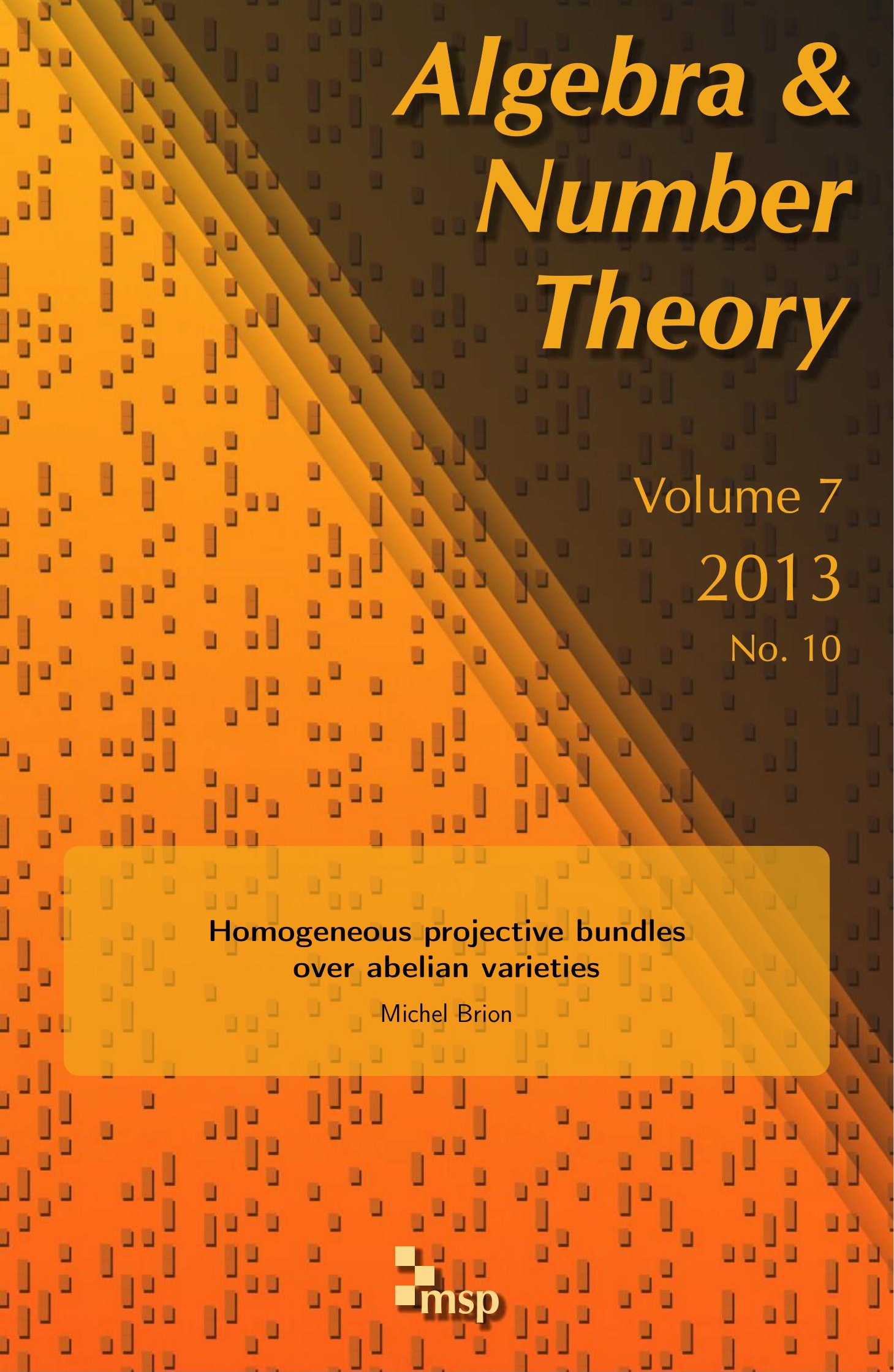




\title{
Homogeneous projective bundles over abelian varieties
}

\author{
Michel Brion
}

\begin{abstract}
We consider projective bundles (or Brauer-Severi varieties) over an abelian variety which are homogeneous, that is, invariant under translation. We describe the structure of these bundles in terms of projective representations of commutative group schemes; the irreducible bundles correspond to Heisenberg groups and their standard representations. Our results extend those of Mukai on semihomogeneous vector bundles, and yield a geometric view of the Brauer group of abelian varieties.
\end{abstract}

\section{Introduction}

The main objects of this article are projective bundles (or Brauer-Severi varieties) over an abelian variety $X$ which are homogeneous, that is, isomorphic to their pull-backs under all translations. Among these bundles, projectivizations of vector bundles are well understood thanks to [Mukai 1978]. Indeed, vector bundles with homogeneous projectivization are exactly the semihomogeneous vector bundles of Mukai. Those that are simple (that is, their global endomorphisms are just scalars) admit several remarkable characterizations; for example, they are all obtained as direct images of line bundles under isogenies. Moreover, every indecomposable semihomogeneous vector bundle is the tensor product of a unipotent bundle and a simple semihomogeneous bundle.

In this article, we obtain somewhat similar statements for the structure of homogeneous projective bundles. We build on the results of [Brion 2012a] about homogeneous principal bundles under an arbitrary algebraic group; here we consider of course the projective linear group $\mathrm{PGL}_{n}$. In loose terms, the approach of our earlier paper reduces the classification of homogeneous bundles to that of commutative subgroup schemes of $\mathrm{PGL}_{n}$. The latter, carried out in Section 2, is based on the classical construction of Heisenberg groups and their irreducible representations.

In Section 3, we introduce a notion of irreducibility for homogeneous projective bundles, which is equivalent to the group scheme of bundle automorphisms being

MSC2010: primary 14K05; secondary 14F22, 14J60, 14L30.

Keywords: abelian varieties, projective bundles, Heisenberg groups, Brauer group. 
finite. (The projectivization of a semihomogeneous vector bundle $E$ is irreducible if and only if $E$ is simple.) We characterize those projective bundles that are homogeneous and irreducible by the vanishing of all the cohomology groups of their adjoint vector bundle (Proposition 3.7). Also, we show that the homogeneous irreducible bundles are classified by the pairs $(H, e)$, where $H$ is a finite subgroup of the dual abelian variety, and $e: H \times H \rightarrow \mathbb{G}_{m}$ a nondegenerate alternating bilinear pairing (Proposition 3.1). Finally, we obtain a characterization of those homogeneous projective bundles that are projectivizations of vector bundles, first in the irreducible case (Proposition 3.10; it states in loose terms that the pairing $e$ originates from a line bundle on $X$ ) and then in the general case (Theorem 3.11).

Irreducible homogeneous projective bundles over an elliptic curve are exactly the projectivizations of indecomposable vector bundles with coprime rank and degree, as follows from the classic work of Atiyah [1957]. But any abelian variety $X$ of dimension at least two admits many homogeneous projective bundles that are not projectivizations of vector bundles. In fact, any class in the Brauer group $\mathrm{Br} X$ is represented by a homogeneous bundle (as shown by [Elencwajg and Narasimhan 1983, Theorem 1] in the setting of complex tori). Also, our approach yields a geometric view of a description of $\operatorname{Br} X$ due to Berkovich [1972]; this is developed in Remark 3.13.

Spaces of algebraically equivalent effective divisors on an arbitrary projective variety afford geometric examples of projective bundles. These spaces are investigated in Section 4 for abelian varieties and curves of genus $g \geq 2$; they turn out to be homogeneous in the former case, but not in the latter.

Finally, in Section 5 we investigate those homogeneous projective bundles that are self-dual, that is, equipped with an isomorphism to their dual bundle; these correspond to principal bundles under the projective orthogonal or symplectic groups. Here the main ingredients are the Heisenberg groups associated to symplectic vector spaces over a field with two elements. Also, we introduce a geometric notion of indecomposability (which differs from the group-theoretic notion of $L$-indecomposability defined in [Balaji et al. 2005]), and obtain a structure result for indecomposable homogeneous self-dual bundles (Proposition 5.9).

Throughout this article, the base field $k$ is algebraically closed, of arbitrary characteristic $p \geq 0$. Most of our results on $\mathbb{P}^{n-1}$-bundles hold under the assumption that $n$ is not a multiple of $p$; indeed, the structure of commutative subgroup schemes of $\mathrm{PGL}_{n}$ is much more complicated when $p$ divides $n$ (see [Levy et al. 2009]). For the same reason, we only consider self-dual projective bundles in characteristic other than 2. It would be interesting to extend our results to "bad" characteristics.

Notation and conventions. We use the book [Demazure and Gabriel 1970] as a general reference for group schemes. Our reference for abelian varieties is [Mumford 1970]; we generally follow its notation. In particular, the group law of an abelian 
variety $X$ is denoted additively and multiplication by an integer $n$ is denoted by $n_{X}$, with kernel $X_{n}$. For any point $x \in X$, we denote by $T_{x}: X \rightarrow X$ the translation $y \mapsto x+y$. The dual abelian variety is denoted by $\widehat{X}$.

\section{Structure of homogeneous projective bundles}

Generalities on projective bundles. Recall that a projective bundle over a variety $X$ is a variety $P$ equipped with a proper flat morphism

$$
f: P \rightarrow X
$$

with fibers at all closed points isomorphic to projective space $\mathbb{P}^{n-1}$ for some integer $n \geq 1$. Then $f$ is a $\mathbb{P}^{n-1}$-bundle for the étale topology (see [Grothendieck 1968a, $\S 8])$.

Also, recall from [loc. cit.] that the $\mathbb{P}^{n-1}$-bundles are in a one-to-one correspondence with the torsors (or principal bundles)

$$
\pi: Y \rightarrow X
$$

under the projective linear group, $\mathrm{PGL}_{n}=\operatorname{Aut}\left(\mathbb{P}^{n-1}\right)$. Specifically, $P$ is the associated bundle $Y \times{ }^{\mathrm{PGL}_{n}} \mathbb{P}^{n-1}$, and $Y$ is the bundle of isomorphisms $X \times \mathbb{P}^{n-1} \rightarrow P$ over $X$. Thus, any representation $\rho: \mathrm{PGL}_{n} \rightarrow \mathrm{GL}(V)$ defines the associated vector bundle $Y \times{ }^{\mathrm{PGL}_{n}} V$ over $X$. The representation of $\mathrm{PGL}_{n}$ in the space $\mathrm{M}_{n}$ of $n \times n$ matrices by conjugation yields a matrix bundle on $X$; its sheaf of local sections is an Azumaya algebra of rank $n^{2}$ over $X$,

$$
\mathscr{A}:=\left(\pi_{*}\left(\mathscr{O}_{Y}\right) \otimes \mathrm{M}_{n}\right)^{\mathrm{PGL}_{n}},
$$

viewed as a sheaf of noncommutative $\mathrm{O}_{X}$-algebras over $\pi_{*}\left(\mathrm{O}_{Y}\right)^{\mathrm{PGL}_{n}}=\mathrm{O}_{X}$. In particular, $\mathscr{A}$ defines a central simple algebra of degree $n$ over the function field $k(X)$. By [Grothendieck 1968a, corollaire 5.11], the assignment $P \mapsto \mathscr{A}$ yields a one-to-one correspondence between $\mathbb{P}^{n-1}$-bundles and Azumaya algebras of rank $n^{2}$. The quotient of $\mathscr{A}$ by $O_{X}$ is the sheaf of local sections of the adjoint bundle ad $P$, the vector bundle associated with the adjoint representation of $\mathrm{PGL}_{n}$ in its Lie algebra $\mathfrak{p g l}_{n}$ (the quotient of the Lie algebra $\mathrm{M}_{n}$ by the scalar matrices). The correspondences between $\mathbb{P}^{n-1}$-bundles, $\mathrm{PGL}_{n}$-torsors, and Azumaya algebras of rank $n^{2}$ preserve morphisms. As a consequence, every morphism of $\mathbb{P}^{n-1}$-bundles is an isomorphism.

There is a natural operation of product on projective bundles: to any $\mathbb{P}^{n_{i}-1}$-bundles $f_{i}: P_{i} \rightarrow X(i=1,2)$ with associated $\mathrm{PGL}_{n_{i}}$-bundles $\pi_{i}: Y_{i} \rightarrow X$, one associates the $\mathbb{P}^{n_{1} n_{2}-1}$-bundle

$$
f: P_{1} P_{2} \rightarrow X
$$


that corresponds to the $\mathrm{PGL}_{n_{1} n_{2}}$-torsor obtained from the $\mathrm{PGL}_{n_{1}} \times \mathrm{PGL}_{n_{2}}$-torsor

$$
\pi_{1} \times \pi_{2}: Y_{1} \times{ }_{X} Y_{2} \rightarrow X
$$

by the extension of structure groups

$$
\operatorname{PGL}_{n_{1}} \times \operatorname{PGL}_{n_{2}}=\operatorname{PGL}\left(k^{n_{1}}\right) \times \operatorname{PGL}\left(k^{n_{2}}\right) \stackrel{\rho}{\rightarrow} \operatorname{PGL}\left(k^{n_{1}} \otimes k^{n_{2}}\right)=\operatorname{PGL}_{n_{1} n_{2}},
$$

where $\rho$ stems from the natural representation $\operatorname{GL}\left(k^{n_{1}}\right) \times \operatorname{GL}\left(k^{n_{2}}\right) \rightarrow \operatorname{GL}\left(k^{n_{1}} \otimes k^{n_{2}}\right)$. So $P_{1} P_{2}$ contains the fibered product $P_{1} \times{ }_{X} P_{2}$; it may be viewed as a global analogue of the Segre product of projective spaces. The corresponding operation on Azumaya algebras is the tensor product (see [Grothendieck 1968a, §8]).

Likewise, any projective bundle $f: P \rightarrow X$ has a dual bundle

$$
f^{*}: P^{*} \rightarrow X
$$

where $P^{*}$ is the same variety as $P$, but the action of $\mathrm{PGL}_{n}$ is twisted by the automorphism arising from the inverse transpose; then $P^{*}=Y \times{ }^{\mathrm{PGL}_{n}}\left(\mathbb{P}^{n-1}\right)^{*}$, where $\left(\mathbb{P}^{n-1}\right)^{*}$ denotes the dual projective space. The Azumaya algebra associated with $P^{*}$ is the opposite algebra $\mathscr{A}^{\mathrm{op}}$. The assignment $P \mapsto P^{*}$ is of course contravariant, and the bidual $P^{* *}$ comes with a canonical isomorphism of bundles $P \stackrel{\cong}{\leftrightarrows} P^{* *}$.

Given a positive integer $n_{1} \leq n$, a $\mathbb{P}^{n_{1}-1}$-subbundle $f_{1}: P_{1} \rightarrow X$ of the $\mathbb{P}^{n-1}$-bundle (1) corresponds to a reduction of structure group of the associated $\mathrm{PGL}_{n}$-torsor (2) to a $\mathrm{PGL}_{n, n_{1}}$-torsor $\pi_{1}: Y_{1} \rightarrow X$, where $\mathrm{PGL}_{n, n_{1}} \subset \mathrm{PGL}_{n}$ denotes the maximal parabolic subgroup that stabilizes a linear subspace $\mathbb{P}^{n_{1}-1}$ of $\mathbb{P}^{n-1}$. Equivalently, the subbundle $P_{1}$ corresponds to a $\mathrm{PGL}_{n}$-equivariant morphism

$$
\gamma: Y \rightarrow \mathrm{PGL}_{n} / \mathrm{PGL}_{n, n_{1}}=\mathrm{Gr}_{n, n_{1}}
$$

(the Grassmannian parametrizing these subspaces). We have $P \cong Y_{1} \times{ }^{\mathrm{PGL}_{n, n_{1}} \mathbb{P}^{n-1}}$ and $P_{1} \cong Y_{1} \times{ }^{\mathrm{PGL}_{n, n_{1}}} \mathbb{P}^{n_{1}-1}$ as bundles over $X$, where $\mathrm{PGL}_{n, n_{1}}$ acts on $\mathbb{P}^{n_{1}-1}$ via its quotient $\mathrm{PGL}_{n_{1}}$.

Given two positive integers $n_{1}$ and $n_{2}$ such that $n_{1}+n_{2}=n$, a decomposition of type $\left(n_{1}, n_{2}\right)$ of the $\mathbb{P}^{n-1}$-bundle (1) consists of two $\mathbb{P}^{n_{i}-1}$-subbundles $f_{i}: P_{i} \rightarrow X$ $(i=1,2)$ which are disjoint (as subvarieties of $P$ ). This corresponds to a reduction of structure group of the $\mathrm{PGL}_{n}$-torsor (2) to a torsor $\pi_{12}: Y_{12} \rightarrow X$ under the maximal Levi subgroup

$$
\mathrm{P}\left(\mathrm{GL}_{n_{1}} \times \mathrm{GL}_{n_{2}}\right)=\mathrm{PGL}_{n, n_{1}} \cap \mathrm{PGL}_{n, n_{2}} \subset \mathrm{PGL}_{n}
$$

that stabilizes two disjoint linear subspaces $\mathbb{P}^{n_{i}-1}$ of $\mathbb{P}^{n-1}(i=1,2)$. Then

$$
P_{i}=Y_{12} \times{ }^{\mathrm{P}\left(\mathrm{GL}_{n_{1}} \times \mathrm{GL}_{n_{2}}\right)} \mathbb{P}^{n_{i}-1}
$$


for $i=1,2$, where $\mathrm{P}\left(\mathrm{GL}_{n_{1}} \times \mathrm{GL}_{n_{2}}\right)$ acts on each $\mathbb{P}^{n_{i}-1}$ via its quotient $\mathrm{PGL}_{n_{i}}$. The decompositions of type $\left(n_{1}, n_{2}\right)$ correspond to the $\mathrm{PGL}_{n}$-equivariant morphisms

$$
\delta: Y \rightarrow \mathrm{PGL}_{n} / \mathrm{P}\left(\mathrm{GL}_{n_{1}} \times \mathrm{GL}_{n_{2}}\right)
$$

to the variety of decompositions.

If the bundle (1) admits no decomposition, then we say, of course, that it is indecomposable. Equivalently, the associated torsor (2) admits no reduction of structure group to a proper Levi subgroup.

When $P$ is the projectivization $\mathbb{P}(E)$ of a vector bundle $E$ over $X$, the subbundles of $P$ correspond bijectively to those of $E$, and the decompositions of $P$ to the splittings $E=E_{1} \oplus E_{2}$ of vector bundles. Also, note that $\mathbb{P}(E) \mathbb{P}(F)=\mathbb{P}(E \otimes F)$ and $\mathbb{P}(E)^{*}=\mathbb{P}\left(E^{*}\right)$, with obvious notation.

Homogeneous projective bundles. From now on, $X$ denotes a fixed abelian variety, $f: P \rightarrow X$ a $\mathbb{P}^{n-1}$-bundle, and $\pi: Y \rightarrow X$ the corresponding $\mathrm{PGL}_{n}$-torsor. Then $P$ is a nonsingular projective variety and $f$ is its Albanese morphism. In particular, $f$ is uniquely determined by the variety $P$.

Since $P$ is complete, its automorphism functor is represented by a group scheme Aut $P$, locally of finite type. Moreover, we have a homomorphism of group schemes

$$
f_{*}: \operatorname{Aut}(P) \rightarrow \operatorname{Aut}(X)
$$

with kernel the subgroup scheme $\operatorname{Aut}_{X}(P) \cong \operatorname{Aut}_{X}^{\mathrm{PGL}_{n}}(Y)$ of bundle automorphisms. Also, $\operatorname{Aut}_{X}(P)$ is affine of finite type, and its Lie algebra is $H^{0}(X, \operatorname{ad}(P))$ (see, for example, [Brion 2011, §4] for these results).

We say that a $\mathbb{P}^{n-1}$-bundle (1) is homogeneous if the image of $f_{*}$ contains the subgroup $X \subset \operatorname{Aut}(X)$ of translations; equivalently, the bundle $P$ is isomorphic to its pull-backs under all translations. This amounts to the vector bundle ad $P$ being homogeneous (see [Brion 2012a, Corollary 2.15]; if $P$ is the projectivization of a vector bundle, this follows alternatively from [Mukai 1978, Theorem 5.8]).

The structure of homogeneous projective bundles is described by the following:

Theorem 2.1. (i) $A \mathbb{P}^{n-1}$-bundle $f: P \rightarrow X$ is homogeneous if and only if there exist an exact sequence of group schemes

$$
1 \longrightarrow H \longrightarrow G \stackrel{\gamma}{\longrightarrow} X \longrightarrow 1,
$$

where $G$ is antiaffine (i.e., $\mathrm{O}(G)=k$ ), and a faithful homomorphism $\rho: H \rightarrow \mathrm{PGL}_{n}$ such that $P$ is the associated bundle $G \times{ }^{H} \mathbb{P}^{n-1} \rightarrow G / H=X$, where $H$ acts on $\mathbb{P}^{n-1}$ via $\rho$.

Then the exact sequence (4) is unique; the group scheme $G$ is smooth, connected, and commutative (in particular, $H$ is commutative), and the projective representation $\rho$ is unique up to conjugacy in $\mathrm{PGL}_{n}$. Moreover, the corresponding 
$\mathrm{PGL}_{n}$-torsor is the associated bundle $G \times{ }^{H} \mathrm{PGL}_{n} \rightarrow X$, and the corresponding Azumaya algebra satisfies

$$
\mathscr{A} \cong\left(\gamma_{*}\left(\mathcal{O}_{G}\right) \otimes \mathrm{M}_{n}\right)^{H}
$$

as a sheaf of algebras over $\gamma_{*}\left(\mathscr{O}_{G}\right)^{H} \cong \mathrm{O}_{X}$.

(ii) For $P$ as in (i), we have an isomorphism

$$
\operatorname{Aut}_{X}(P) \cong \mathrm{PGL}_{n}^{H},
$$

the right-hand side being the centralizer of $H$ in $\mathrm{PGL}_{n}$. As a consequence,

$$
H^{0}(X, \operatorname{ad}(P))=\mathfrak{p g l}_{n}^{H} .
$$

(iii) The homogeneous projective subbundles of $P$ are exactly the bundles $G \times{ }^{H} S \rightarrow$ $X$, where $S \subset \mathbb{P}^{n-1}$ is an $H$-stable linear subspace.

(iv) Any decomposition of $P$ consists of homogeneous subbundles.

Proof. Part (i) follows readily from Theorem 3.1 of [Brion 2012a], and (ii) from Proposition 3.6 of the same reference.

(iii) Let $f_{1}: P_{1} \rightarrow X$ be a projective subbundle, and consider the corresponding reduction of structure group of the $\mathrm{PGL}_{n}$-torsor $Y$ to a $\mathrm{PGL}_{n, n_{1}}$-torsor $\pi_{1}: Y_{1} \rightarrow X$. If $f_{1}$ is homogeneous, then again by [Brion 2012a, Theorem 3.1], we have a $\mathrm{PGL}_{n, n_{1}}$-equivariant isomorphism

$$
Y_{1} \cong G_{1} \times{ }^{H_{1}} \mathrm{PGL}_{n, n_{1}}
$$

for some exact sequence $0 \rightarrow H_{1} \rightarrow G_{1} \rightarrow X \rightarrow 0$ with $G_{1}$ antiaffine, and some faithful homomorphism $\rho_{1}: H_{1} \rightarrow \mathrm{PGL}_{n, n_{1}}$. Thus,

$$
Y \cong Y_{1} \times \mathrm{PGL}_{n, n_{1}} \mathrm{PGL}_{n} \cong G_{1} \times{ }^{H_{1}} \mathrm{PGL}_{n}
$$

equivariantly for the action of $\mathrm{PGL}_{n}$. By the uniqueness in (i), it follows that $G_{1}=G$ and $H_{1}=H$; hence $P_{1}=G \times{ }^{H} S$ for some $H$-stable linear subspace $S \subset \mathbb{P}^{n-1}$.

Conversely, any $\mathrm{H}$-stable linear subspace obviously yields a homogeneous projective subbundle.

(iv) A decomposition of $P$ of type $\left(n_{1}, n_{2}\right)$ corresponds to a $\mathrm{PGL}_{n}$-equivariant morphism $\delta: Y \rightarrow \mathrm{PGL}_{n} / \mathrm{P}\left(\mathrm{GL}_{n_{1}} \times \mathrm{GL}_{n_{2}}\right)$. Since the variety $\mathrm{PGL}_{n} / \mathrm{P}\left(\mathrm{GL}_{n_{1}} \times \mathrm{GL}_{n_{2}}\right)$ is affine, the corresponding reduction of structure group $\pi_{12}: Y_{12} \rightarrow X$ is homogeneous by [loc. cit., Proposition 2.8]. Thus, the associated bundles $P_{1}$ and $P_{2}$ are homogeneous as well. 
Remark 2.2. Let $P_{i}(i=1,2)$ be homogeneous bundles corresponding to extensions $1 \rightarrow H_{i} \rightarrow G_{i} \rightarrow X \rightarrow 1$ and projective representations $\rho_{i}: H_{i} \rightarrow$ PGL $_{n_{i}}$. Then the $\mathrm{PGL}_{n_{1} n_{2}}$-torsor that corresponds to $P_{1} P_{2}$ is the associated bundle

$$
\left(G_{1} \times{ }_{X} G_{2}\right) \times{ }^{H_{1} \times H_{2}} \mathrm{PGL}_{n_{1} n_{2}} \longrightarrow\left(G_{1} \times{ }_{X} G_{2}\right) /\left(H_{1} \times H_{2}\right)=X,
$$

where the homomorphism $H_{1} \times H_{2} \rightarrow$ PGL $_{n_{1} n_{2}}$ is given by the tensor product $\rho_{1} \otimes \rho_{2}$. Thus, $P_{1} P_{2}$ is the homogeneous bundle classified by the extension $1 \rightarrow$ $H \rightarrow G \rightarrow X \rightarrow 1$, where $G \subset G_{1} \times{ }_{X} G_{2}$ denotes the largest antiaffine subgroup and $H=\left(H_{1} \times H_{2}\right) \cap G$, and by the projective representation $\left.\left(\rho_{1} \otimes \rho_{2}\right)\right|_{H}$.

As a consequence, the $m$-th power $P^{m}$ corresponds to the same extension as $P$ and to the $m$-th tensor power of its projective representation. Likewise, the dual of a homogeneous bundle is the homogeneous bundle associated with the same extension and with the dual projective representation.

The antiaffine algebraic groups are classified in [Brion 2009] and independently [Sancho de Salas and Sancho de Salas 2009], and the antiaffine extensions (4) in [Brion 2012a, §3.3]. We now describe the other ingredients of Theorem 2.1, that is, the commutative subgroup schemes $H \subset \mathrm{PGL}_{n}$ up to conjugacy. Every such subgroup scheme has a unique decomposition

$$
H=H_{u} \times H_{s},
$$

where $H_{u}$ is unipotent and $H_{s}$ is diagonalizable. Thus, $H_{s}$ sits in an exact sequence

$$
1 \rightarrow H_{s}^{0} \rightarrow H_{s} \rightarrow F \rightarrow 1,
$$

where $H_{s}^{0}$ is a connected diagonalizable group scheme (the neutral component of $H_{s}$ ), and the group of components $F$ is finite, diagonalizable, and of order prime to $p$ (in particular, $F$ is smooth); this exact sequence is unique and splits noncanonically. In turn, $H_{s}^{0}$ is an extension of a finite diagonalizable group scheme of order a power of $p$, by a torus (the reduced neutral component); this extension is also unique and splits noncanonically.

Denote by $\widetilde{H} \subset \mathrm{GL}_{n}$ the preimage of $H \subset \mathrm{PGL}_{n}$. This yields a central extension

$$
1 \rightarrow \mathbb{G}_{m} \rightarrow \widetilde{H} \rightarrow H \rightarrow 1,
$$

where the multiplicative group $\mathbb{G}_{m}$ is viewed as the group of invertible scalar matrices. We say that $\widetilde{H}$ is the theta group of $H$, and define similarly $\widetilde{H}_{u}, \widetilde{H}_{s}$, and $\widetilde{H}_{s}^{0}$ (the latter is the neutral component of $\widetilde{H}_{s}$ ).

Given two $S$-valued points $\tilde{x}$ and $\tilde{y}$ of $\tilde{H}$, where $S$ denotes an arbitrary scheme, the commutator $\tilde{x} \tilde{y} \tilde{x}^{-1} \tilde{y}^{-1}$ is a $S$-valued point of $\mathbb{G}_{m}$ and depends only on the images of $\tilde{x}$ and $\tilde{y}$ in $H$. This defines a morphism

$$
e: H \times H \rightarrow \mathbb{G}_{m},
$$


which is readily seen to be bilinear (that is, we have $e(x y, z)=e(x, z) e(y, z)$ and $e(x, y z)=e(x, z) e(y, z)$ for all $S$-valued points $x, y, z$ of $H)$ and alternating (that is, $e(x, x)=1$ for all $x)$. We say that $e$ is the commutator pairing of the extension (8).

Note that the dual bundle $P^{*}$ has pairing $e^{-1}$; moreover, the power $P^{m}$, where $m$ is a positive integer, has pairing $e^{m}$.

The center $Z(\widetilde{H})$ sits in an exact sequence of group schemes

$$
1 \rightarrow \mathbb{G}_{m} \rightarrow Z(\widetilde{H}) \rightarrow H^{\perp} \rightarrow 1,
$$

where the $S$-valued points of $H^{\perp}$ are those points of $H$ such that $e(x, y)=1$ for all $S^{\prime}$-valued points $y$ of $H$ and all schemes $S^{\prime}$ over $S$. In particular, $\widetilde{H}$ is commutative if and only if $e=1$.

We now show that the obstruction for being the projectivization of a homogeneous vector bundle is just the commutator pairing. The obstruction for being the projectivization of an arbitrary vector bundle will be determined in Theorem 3.11.

Proposition 2.3. With the above notation, the following conditions are equivalent:

(i) $P$ is the projectivization of a homogeneous vector bundle.

(ii) The extension (8) splits.

(iii) $e=1$.

Proof. (i) $\Longrightarrow$ (ii) By [Brion 2012a, Theorem 3.1], any homogeneous vector bundle $E$ of rank $n$ over $X$ is of the form $G \times{ }^{H} k^{n} \rightarrow G / H=X$ for some antiaffine extension $1 \rightarrow H \rightarrow G \rightarrow X \rightarrow 1$ and some faithful representation $\sigma: H \rightarrow \mathrm{GL}_{n}$. Since $H$ is commutative, $k^{n}$ contains eigenvectors of $H$; thus, twisting $\sigma$ by a character of $H$ (which does not change the projectivization $\mathbb{P}(E)$ ), we may assume that $k^{n}$ contains nonzero fixed points of $H$. Then $\sigma$ defines a faithful projective representation $\rho: H \rightarrow \mathrm{PGL}_{n}$. Hence $G$ and $\rho$ are the data associated with the homogeneous projective bundle $\mathbb{P}(E) \rightarrow X$, and $\sigma$ splits the extension (8).

(ii) $\Longrightarrow$ (i) Any splitting of that extension yields a homomorphism $\sigma: H \rightarrow \mathrm{GL}_{n}$ that lifts $\rho$. Then the associated bundle $G \times{ }^{H} k^{n} \rightarrow X$ is a homogeneous vector bundle with projectivization $P$.

(ii) $\Longleftrightarrow$ (iii) The forward implication is obvious. Conversely, if $e=1$, then $\widetilde{H}$ is commutative. It follows that $\widetilde{H} \cong U \times \widetilde{H}_{s}$, where the unipotent part $U$ is isomorphic to $H_{u}$ via the homomorphism $\widetilde{H} \rightarrow H$, and $\widetilde{H}_{s}$ sits in an exact sequence of diagonalizable group schemes $1 \rightarrow \mathbb{G}_{m} \rightarrow \widetilde{H}_{s} \rightarrow H_{s} \rightarrow 1$. But every such sequence splits, since so does the dual exact sequence of character groups.

Next, we obtain a very useful structure result for $H$ under the assumption that $n$ is not divisible by the characteristic:

Proposition 2.4. Keep the above notation, and assume that $(n, p)=1$. 
(i) The extension $1 \rightarrow \mathbb{G}_{m} \rightarrow \widetilde{H}_{u} \rightarrow H_{u} \rightarrow 1$ has a unique splitting, and the corresponding lift of $H_{u}$ (that we still denote by $H_{u}$ ) is central in $\widetilde{H}$. Also, the extension $1 \rightarrow \mathbb{G}_{m} \rightarrow \widetilde{H}_{s}^{0} \rightarrow H_{s}^{0} \rightarrow 1$ splits noncanonically and $\widetilde{H}_{s}^{0}$ is central in $\widetilde{H}$.

(ii) We have canonical decompositions of group schemes

$$
\widetilde{H}=H_{u} \times \widetilde{H}_{s}, \quad Z(\widetilde{H})=H_{u} \times Z\left(\widetilde{H}_{s}\right) .
$$

Moreover, $Z\left(\widetilde{H}_{s}\right)$ is diagonalizable and sits in an exact sequence

$$
1 \rightarrow \widetilde{H}_{s}^{0} \rightarrow Z\left(\widetilde{H}_{s}\right) \rightarrow F^{\perp} \rightarrow 1
$$

which splits noncanonically.

(iii) The commutator pairing e factors through a bilinear alternating morphism

$$
e_{F}: F \times F \rightarrow \mathbb{G}_{m} .
$$

Proof. Since any commutator has determinant 1, we see that $e$ takes values in the subgroup scheme $\mu_{n}=\mathbb{G}_{m} \cap \mathrm{SL}_{n}$ of $n$-th roots of unity. In other terms, $e$ factors through the pairing

$$
\text { se }: H \times H \rightarrow \mu_{n}
$$

defined by the central extension

$$
1 \rightarrow \mu_{n} \rightarrow S \tilde{H} \rightarrow H \rightarrow 1
$$

where $S \widetilde{H}:=H \cap \mathrm{SL}_{n}$. Note that $\mu_{n}$ is smooth by our assumption on $n$. Moreover, se restricts trivially to $n H \times H$, where $n H$ denotes the image of the multiplication by $n$ in the commutative group scheme $H$.

We claim that $H_{u} \subset n H$. This is clear if $p=0$, since $H_{u}$ is then isomorphic to the additive group of a vector space. If $p \geq 1$, then the commutative unipotent group scheme $H_{u}$ is killed by some power of $p$. Using again the assumption that $(n, p)=1$, it follows that $H_{u}=n H_{u} \subset n H$.

By that claim, se restricts trivially to $H_{u} \times H$, and hence $\widetilde{H}_{u} \subset Z(\widetilde{H})$; in particular, $\widetilde{H}_{u}$ is commutative. Thus, $\widetilde{H}_{u} \cong H_{u} \times \mathbb{G}_{m}$; this proves the assertion about $H_{u}$.

We already saw that the extension $1 \rightarrow \mathbb{G}_{m} \rightarrow \widetilde{H}_{s}^{0} \rightarrow H_{s}^{0} \rightarrow 1$ splits. Also, $H_{s}^{0} \cong T \times E$, where $T$ is a torus (the reduced neutral component), and $E$ is a finite group scheme killed by some power of $p$. As above, it follows that $H_{s}^{0} \subset n H$, and that $\widetilde{H}_{s}^{0}$ is central in $\widetilde{H}$. This completes the proof of (i).

The decompositions in (ii) are direct consequences of (i). The assertion on $Z\left(\widetilde{H}_{s}\right)$ follows from the exact sequence $1 \rightarrow \widetilde{H}_{s}^{0} \rightarrow \widetilde{H}_{s} \rightarrow F \rightarrow 1$, since $\widetilde{H}_{s}^{0} \subset Z\left(\widetilde{H}_{s}\right)$. Finally, (iii) also follows readily from (i). 
Remark 2.5. With the notation and assumptions of Proposition 2.4, the group scheme $\operatorname{Aut}_{X}(P)$ is smooth, as follows from the isomorphism (6) together with [Herpel 2013, Theorem 1.1]. Moreover, Aut $P$ is smooth as well: indeed, we have an exact sequence of group schemes

$$
1 \longrightarrow \operatorname{Aut}_{X}(P) \longrightarrow \operatorname{Aut}(P) \stackrel{f_{*}}{\longrightarrow} \operatorname{Aut}_{P}(X) \longrightarrow 1,
$$

where $\operatorname{Aut}_{P}(X)$ is a subgroup scheme of Aut $X$ containing the group $X$ of translations. Since $\operatorname{Aut}(X)=X \ltimes \operatorname{Aut}_{\mathrm{gp}}(X)$, where the group scheme of automorphisms of algebraic groups $\operatorname{Aut}_{\mathrm{gp}}(X)$ is étale (possibly infinite), it follows that $\operatorname{Aut}_{P}(X)$ is smooth, and hence so is Aut $X$.

Nondegenerate theta groups. As in the above subsection, we consider a commutative subgroup scheme $H \subset \mathrm{PGL}_{n}$ and the associated theta group $\widetilde{H} \subset \mathrm{GL}_{n}$; we assume that $(n, p)=1$.

We say that $\widetilde{H}$ is nondegenerate if $Z(\widetilde{H})=\mathbb{G}_{m}$. By Proposition 2.4, this is equivalent to the assertions that $H$ is a finite commutative group of order prime to $p$, and the homomorphism

$$
\epsilon: H \rightarrow \mathscr{X}(H), \quad x \mapsto(y \mapsto e(x, y))
$$

is faithful, where $\mathscr{X}(H):=\operatorname{Hom}_{\mathrm{gp}}\left(H, \mathbb{G}_{m}\right)$ denotes the character group of $H$. It follows that $\epsilon$ is an isomorphism.

We now recall from [Mumford 1966, §1] the structure of nondegenerate theta groups. Choose a subgroup $K \subset H$ that is totally isotropic for the commutator pairing $e$, and maximal with this property. Then

$$
\widetilde{H} \cong \mathbb{G}_{m} \times K \times \mathscr{X}(K),
$$

where the group law on the right-hand side is given by

$$
(t, x, \chi) \cdot\left(t^{\prime}, x^{\prime}, \chi^{\prime}\right)=\left(t t^{\prime} \chi^{\prime}(x), x+x^{\prime}, \chi+\chi^{\prime}\right),
$$

the group laws on $K$ and $\mathscr{L}(K)$ being denoted additively. Such a group is called the Heisenberg group associated with the finite group $K$; we denote it by $\mathscr{H}(K)$ and identify the group $K(\operatorname{resp} . \mathscr{L}(K))$ with its lift $\{1\} \times K \times\{0\}(\operatorname{resp} .\{1\} \times\{0\} \times \mathscr{L}(K))$ in $\mathscr{H}(K)$.

Also, recall that $\mathscr{H}(K)$ has a unique irreducible representation on which $\mathbb{G}_{m}$ acts via $t \mapsto t$ id: the standard representation (also called the $S$ chrödinger representation) in the space $\mathcal{O}(K)$ of functions on $K$ with values in $k$, on which $\widetilde{H}$ acts via

$$
((t, x, \chi) \cdot f)(y):=t \chi(y) f(x+y) .
$$

The corresponding commutator pairing $e$ is given by

$$
e\left((x, \chi),\left(x^{\prime}, \chi^{\prime}\right)\right):=\chi^{\prime}(x) \chi\left(x^{\prime}\right)^{-1} \text {. }
$$


In particular, the standard representation $W(K)$ contains a unique line of $K$-fixed points and has dimension $n=\#(K)$; moreover, the group $H$ is killed by $n$ and has order $n^{2}$. Any finite-dimensional representation $V$ of $\mathscr{H}(K)$ on which $\mathbb{G}_{m}$ acts by scalar multiplication is a direct sum of $m$ copies of $W(K)$, where $m:=\operatorname{dim}\left(V^{K}\right)$. Such a representation is called of weight 1.

For later use, we record the following result, which is well-known in the setting of theta structures on ample line bundles over complex abelian varieties (see [Birkenhake and Lange 2004, Lemma 6.6.6 and Exercise 6.10.14]):

Lemma 2.6. Assume that $(n, p)=1$ and let $\widetilde{H} \subset \mathrm{GL}_{n}$ be a nondegenerate theta group.

(i) The algebra $\mathrm{M}_{n}$ has a basis $\left(u_{h}\right)_{h \in H}$ such that every $u_{h}$ is an eigenvector of $H$ (acting by conjugation) with weight $\epsilon(h)$, and

$$
u_{x, \chi} u_{x^{\prime}, \chi^{\prime}}=\chi^{\prime}(x) u_{x+x^{\prime}, \chi+\chi^{\prime}}
$$

for all $h=(x, \chi)$ and $h^{\prime}=\left(x^{\prime}, \chi^{\prime}\right)$ in $H=K \times \mathscr{L}(K)$. In particular, the representation of $H$ in $\mathrm{M}_{n}$ by conjugation is isomorphic to the regular representation.

(ii) The centralizers of $\widetilde{H}$ in $\mathrm{GL}_{n}$ and of $H$ in $\mathrm{PGL}_{n}$ satisfy

$$
\mathrm{GL}_{n}^{\widetilde{H}}=\mathbb{G}_{m}, \quad \mathrm{PGL}_{n}^{H}=H .
$$

Moreover, the normalizers sit in exact sequences

$$
\begin{aligned}
1 & \rightarrow \mathbb{G}_{m} \rightarrow N_{\mathrm{GL}_{n}}(\widetilde{H}) \rightarrow N_{\mathrm{PGL}_{n}}(H) \rightarrow 1, \\
1 & \rightarrow H \rightarrow N_{\mathrm{PGL}_{n}}(H) \rightarrow \operatorname{Aut}(H, e) \rightarrow 1 .
\end{aligned}
$$

Also, we have an isomorphism

$$
\operatorname{Aut}^{\mathbb{G}_{m}}(\widetilde{H}) \cong N_{\mathrm{PGL}_{n}}(H) .
$$

Proof. (i) We may view $H$ as a subset of $\mathrm{M}_{n}$ via $(x, \chi) \mapsto u_{x, \chi}:=(1, x, \chi) \in \widetilde{H}$ $\subset \mathrm{GL}_{n}$. Then the assertions follow readily from (13) for the group law of $\widetilde{H}$.

(ii) By Schur's lemma, we have $\mathrm{GL}_{n}^{\widetilde{H}}=\mathbb{G}_{m}$; this yields the first exact sequence.

In view of (i), the fixed points of $H$ acting on $\mathbb{P}\left(M_{n}\right)$ by conjugation are exactly the points of $H \subset \mathrm{PGL}_{n}$; thus, $\mathrm{PGL}_{n}^{H}=H$. To obtain the second exact sequence, it suffices to show that the image in Aut $H$ of $N_{\mathrm{PGL}_{n}}(H)$ equals $\operatorname{Aut}(H, e)$. But if $g \in \mathrm{PGL}_{n}$ normalizes $H$, then one readily checks that the conjugation $\left.\operatorname{Int}(g)\right|_{H}$ preserves the pairing $e$. Conversely, let $g \in \operatorname{Aut}(H, e)$; then composing the inclusion $\rho: H \rightarrow \mathrm{PGL}_{n}$ with $g$, we obtain a projective representation $\rho_{g}$ with the same commutator pairing. Thus, $\rho_{g}$ lifts to a representation $\tilde{\rho}_{g}: \widetilde{H} \rightarrow \mathrm{GL}_{n}$ which is isomorphic to the standard representation. It follows that $g$ extends to the conjugation by some $\tilde{g} \in \mathrm{GL}_{n}$ that normalizes $H$. 
The isomorphism (14) follows similarly from the fact that the standard representation is the unique irreducible representation of weight 1.

Returning to an arbitrary theta group $\widetilde{H} \subset \mathrm{GL}_{n}$, we now describe the representation of $\widetilde{H}$ in $k^{n}=: V$. Consider the decomposition

$$
V=\bigoplus_{\lambda} V_{\lambda}
$$

into weight spaces of the diagonalizable group $Z\left(\widetilde{H}_{s}\right)$, where $\lambda$ runs over the characters of weight 1 of that group (those that restrict to the identity character of $\left.\mathbb{G}_{m}\right)$. By Proposition 2.4, each $V_{\lambda}$ is stable under $\widetilde{H}$.

Proposition 2.7. With the above notation, each quotient $\widetilde{H}_{S} / \operatorname{ker}(\lambda)$ is isomorphic to the Heisenberg group $\mathscr{H}\left(K / F^{\perp}\right)$, where $K$ denotes a maximal totally isotropic subgroup scheme of $F$ relative to $e_{F}$.

Moreover, we have an isomorphism of representations of $\widetilde{H} \cong H_{u} \times \widetilde{H}_{s}$ :

$$
V_{\lambda} \cong U_{\lambda} \otimes W\left(K / F^{\perp}\right),
$$

where $U_{\lambda}$ is a representation of $H_{u}$ and $W\left(K / F^{\perp}\right)$ is the standard representation of $\widetilde{H}_{s} / \operatorname{ker}(\lambda)$.

Proof. Note that $\lambda$ yields a splitting of (10), and an isomorphism $Z\left(\widetilde{H}_{s}\right) / \operatorname{ker}(\lambda) \cong$ $\mathbb{G}_{m}$. Also, $\widetilde{H}_{s} / Z\left(\widetilde{H}_{s}\right) \cong \widetilde{H} / Z(\widetilde{H}) \cong F / F^{\perp}$ by Proposition 2.4 . Thus, the exact sequence

$$
1 \rightarrow Z\left(\widetilde{H}_{s}\right) / \operatorname{ker}(\lambda) \rightarrow \widetilde{H}_{s} / \operatorname{ker}(\lambda) \rightarrow \widetilde{H}_{s} / Z\left(\widetilde{H}_{s}\right) \rightarrow 1
$$

may be identified with the central extension

$$
1 \rightarrow \mathbb{G}_{m} \rightarrow \widetilde{H}_{s} / \operatorname{ker}(\lambda) \rightarrow F / F^{\perp} \rightarrow 1,
$$

and the corresponding commutator pairing is induced by $e_{F}$. This shows that $\widetilde{H}_{S} / \operatorname{ker}(\lambda)$ is a nondegenerate theta group. Now the first assertion follows from the structure of these groups.

Also, $V_{\lambda}$ is a representation of $\widetilde{H}_{S} / \operatorname{ker}(\lambda)$ on which the center $\mathbb{G}_{m}$ acts with weight 1 , and hence a direct sum of copies of the standard representation. This implies the second assertion in view of Proposition 2.4 again.

Corollary 2.8. With the above notation, the representation of $\widetilde{H}$ in $V$ is an iterated extension of irreducible representations of the same dimension,

$$
d:=\left[K: F^{\perp}\right]=\sqrt{\left[F: F^{\perp}\right]}=\sqrt{\left[H: H^{\perp}\right]} .
$$

In particular, $n$ is a multiple of $d$, with equality if and only if $\widetilde{H}$ is a Heisenberg group acting via its standard representation. 
We say that $d$ is the homogeneous index of the bundle (1); this is the minimal rank of a homogeneous subbundle of $P$ in view of Theorem 2.1. (One can show that the homogeneous index of $P$ is a multiple of the index of the associated central simple algebra over $k(X)$.) Note that $F / F^{\perp}$ is killed by $d$, and hence $e_{F}^{d}=1$. In view of Proposition 2.3, it follows that the $d$-th power $P^{d}$ is the projectivization of a homogeneous vector bundle.

Proposition 2.9. With the notation and assumptions of Proposition 2.4, the following assertions are equivalent for a homogeneous $\mathbb{P}^{n-1}$-bundle $f: P \rightarrow X$ :

(i) $P$ is indecomposable.

(ii) The associated representation $\tilde{\rho}: \widetilde{H} \rightarrow \mathrm{GL}_{n}$ is indecomposable.

(iii) $\widetilde{H}_{s}$ is a Heisenberg group and $V \cong U \otimes W$ as representations of $H \cong H_{u} \times \widetilde{H}_{s}$, where $U$ is an indecomposable representation of $H_{u}$ and $W$ is the standard representation of $\widetilde{H}_{S}$.

(iv) The neutral component $\operatorname{Aut}_{X}^{0}(P)$ is unipotent.

Proof. (i) $\Longleftrightarrow$ (ii) The forward implication is obvious, and the converse follows from Theorem 2.1(iv).

(ii) $\Longleftrightarrow$ (iii) This is a direct consequence of Proposition 2.7.

(iii) $\Longrightarrow$ (iv) Since $(n, p)=1$, we have $\mathrm{M}_{n}=k$ id $\oplus \mathfrak{p g l}_{n}$ as representations of $\mathrm{PGL}_{n}$ acting by conjugation. In view of (7), this yields

$$
\text { Lie } \operatorname{Aut}_{X}(P)=\mathrm{M}_{n}^{H} / k \mathrm{id}=\operatorname{End}^{H}(U \otimes W) / k \text { id . }
$$

Moreover, End ${ }^{H}(U \otimes W) \cong$ End $^{H_{u}}(U)$ by Schur's lemma, and hence

$$
\text { Lie } \operatorname{Aut}_{X}(P) \cong \operatorname{End}^{H_{u}}(U) / k \text { id . }
$$

This isomorphism of Lie algebras arises from the natural homomorphism

$$
\mathrm{GL}(U)^{H_{u}} / \mathbb{G}_{m} \text { id } \rightarrow \operatorname{Aut}_{X}(P) .
$$

Since $\operatorname{Aut}_{X}(P)$ is smooth (Remark 2.5), we see that its neutral component is a quotient of $\operatorname{GL}(U)^{H_{u}} / \mathbb{G}_{m}$ id. But the latter group is unipotent, since $U$ is indecomposable.

(iv) $\Longrightarrow$ (iii) Observe that the weight space decomposition (15) is trivial: otherwise, Aut $_{X}(P)$ contains a copy of $\mathbb{G}_{m}$ that fixes some weight space pointwise and acts by scalar multiplication on all the other weight spaces. Thus, $V \cong U \otimes W$, where $W$ is irreducible. Moreover, $U$ is indecomposable; otherwise, $\operatorname{Aut}_{X}(P)$ contains a copy of $\mathbb{G}_{m}$ by the above argument.

Remarks 2.10. (1) The results of this subsection do not extend readily to the case where $p$ divides $n$ : for instance, there exists a nondegenerate theta group $\widetilde{H} \subset \mathrm{GL}_{p}$ 
with $H$ unipotent and local. Consider indeed the group scheme $\alpha_{p}$ (the kernel of the $p$-th power map of $\mathbb{G}_{a}$ ) and the duality pairing

$$
u: \alpha_{p} \times \alpha_{p} \rightarrow \mathbb{G}_{m}, \quad(x, y) \longmapsto \sum_{i=0}^{p-1} \frac{x^{i}}{i !} .
$$

This yields a bilinear alternating pairing $e$ on $H:=\alpha_{p} \times \alpha_{p}$ via

$$
e\left((x, y),\left(x^{\prime}, y^{\prime}\right)\right):=u\left(x, y^{\prime}\right) u\left(x^{\prime}, y\right)^{-1} .
$$

Then we may take for $\widetilde{H}$ the associated Heisenberg group scheme (with $K=\alpha_{p} \times\{0\}$ and $\left.\mathscr{L}(K)=\{0\} \times \alpha_{p}\right)$, equipped with its standard representation in $\mathcal{O}\left(\alpha_{p}\right) \cong k^{p}$.

Note that the above group scheme $H$ is contained in an abelian surface, the product of two supersingular elliptic curves. More generally, any finite commutative group scheme is contained in some abelian variety (see [Oort 1966, §15.4]).

(2) For an arbitrary homogeneous projective bundle $P$, each representation $U_{\lambda}$ (with the notation of Proposition 2.7) is a direct sum of indecomposable representations with multiplicities; moreover, these indecomposable summands and their multiplicities are uniquely determined up to reordering, in view of the KrullSchmidt theorem. Thus, the representation of $\widetilde{H}$ in $V$ decomposes into a direct sum (with multiplicities) of tensor products $U \otimes W$, where $U$ is an indecomposable representation of $H_{u}$ and $W$ is an irreducible representation of $\widetilde{H}_{s}$.

Let $L \subset \mathrm{PGL}_{n}$ denote the stabilizer of such a decomposition. Then $L$ is a Levi subgroup, uniquely determined up to conjugation; moreover, the $\mathrm{PGL}_{n}$-torsor $\pi: Y \rightarrow X$ admits a reduction of structure group to an $L$-torsor $\pi_{L}: Y_{L} \rightarrow X$. Arguing as in the proof that (iii) implies (iv) above, one may check that the natural homomorphism $Z(L) \rightarrow \operatorname{Aut}_{X}^{L}\left(Y_{L}\right)$ (where $Z(L)$ denotes the center of $L$, and $\operatorname{Aut}_{X}^{L}\left(Y_{L}\right)$ the group of bundle automorphisms of $Y_{L}$ ) yields an isomorphism of the reduced neutral component $Z(L)_{\text {red }}^{0}$ to a maximal torus of $\operatorname{Aut}_{X}^{L}\left(Y_{L}\right)$. Thus, the torsor $\pi_{L}: Y_{L} \rightarrow X$ is L-indecomposable in the sense of Definition 2.1 of [Balaji et al. 2005]. Moreover, this torsor is the unique reduction of $\pi: P \rightarrow X$ to an $L$-indecomposable torsor for a Levi subgroup, by Theorem 3.4 of the same reference (the latter result is obtained there in characteristic zero, and generalized to arbitrary characteristics in [Balaji et al. 2006b]; see also [Balaji et al. 2006a]).

Conversely, the equivalence of statements (i) and (iv) above follows from the results of [Balaji et al. 2005; 2006b] in view of the smoothness of $\operatorname{Aut}_{X}(P)$.

\section{Irreducible bundles}

Throughout this section, we consider $\mathbb{P}^{n-1}$-bundles $f: P \rightarrow X$, and call them bundles for simplicity; we still assume that $(n, p)=1$. 
Structure and characterizations. We say that a homogeneous bundle $P$ is irreducible if so is the projective representation $\rho: H \rightarrow \mathrm{PGL}_{n}$ associated with $P$ via Theorem 2.1. By Proposition 2.7, this means that the theta group $\widetilde{H}$ is a Heisenberg group acting on $k^{n}$ via its standard representation.

We now parametrize the irreducible homogeneous bundles, and describe the corresponding Azumaya algebras as well as the adjoint bundles and automorphism groups:

Proposition 3.1. (i) The irreducible homogeneous $\mathbb{P}^{n-1}$-bundles are classified by the pairs $(H, e)$, where $H \subset X_{n}$ is a subgroup of order $n^{2}$ and $e: H \times H \rightarrow \mathbb{G}_{m}$ is a nondegenerate alternating pairing. In particular, such bundles exist for any given $n$, and they form only finitely many isomorphism classes.

(ii) For the bundle P corresponding to $(H, e)$, the associated Azumaya algebra $A$ admits a grading by the group $H$, namely,

$$
\mathscr{A} \cong \bigoplus_{\mathscr{L} \in H} \mathscr{L}
$$

where each element of $H \subset \widehat{X}$ is viewed as an invertible sheaf on $X$. In particular, we have a decomposition

$$
\operatorname{ad}(P) \cong \bigoplus_{\mathscr{L} \in H, \mathscr{L} \neq 0} \mathscr{L}
$$

(iii) For $P$ as in (ii), we have $\operatorname{Aut}_{X}(P) \cong H$. Moreover, the neutral component $\operatorname{Aut}^{0}(P)$ is the extension of $X$ by $H$, dual to the inclusion $\mathscr{X}(H) \cong H \subset \widehat{X}$, and $\operatorname{Aut}(P) / \operatorname{Aut}^{0}(P)$ is isomorphic to the subgroup of $\operatorname{Aut}_{\mathrm{gp}}(X) \cong \operatorname{Aut}_{\mathrm{gp}}(\widehat{X})$ that preserves $H$ and $e$.

Proof. (i) By the results of Section 2, the irreducible homogeneous bundles are classified by the pairs consisting of an isogeny $1 \rightarrow H \rightarrow G \rightarrow X \rightarrow 1$ and a nondegenerate alternating pairing $e$ on $H$; then $e$ provides an isomorphism of $H$ with its character group. The assertion now follows from duality of isogenies.

(ii) This follows from the isomorphism of $O_{X}$-algebras (5) together with the isomorphism of $\mathscr{O}_{X}-H$-algebras $\gamma_{*}\left(\mathscr{O}_{G}\right) \cong \bigoplus_{\mathscr{L} \in \mathscr{L}(H)} \mathscr{L}$ and with the decomposition $\mathrm{M}_{n} \cong \bigoplus_{h \in H} k u_{h}$ obtained in Lemma 2.6(i).

(iii) Combining the isomorphism (6) and Lemma 2.6(ii), we see that the natural map $H \rightarrow \operatorname{Aut}_{X}(P)$ is an isomorphism. In view of the commutative diagram with exact rows

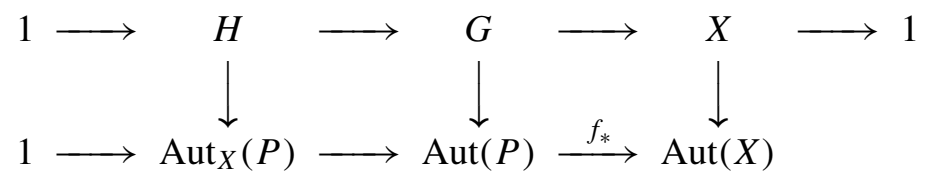


and of the isomorphism $\operatorname{Aut}(X) \cong X \ltimes \operatorname{Aut}_{\mathrm{gp}}(X)$, where $\operatorname{Aut}_{\mathrm{gp}}(X)$ is étale, it follows that the natural map $G \rightarrow \operatorname{Aut}^{0}(P)$ is an isomorphism as well. The structure of $\operatorname{Aut}(P) / \operatorname{Aut}^{0}(P)$ follows from Theorem 2.1 together with Lemma 2.6(ii).

Remark 3.2. Recall from [Mumford 1966, §1] that every finite commutative group $H$ of order prime to $p$, equipped with a nondegenerate alternating pairing $e$, admits a decomposition

$$
H=H_{n_{1}} \times \cdots \times H_{n_{r}}, \quad e=\left(e_{d_{1}}, \ldots, e_{d_{r}}\right),
$$

such that

$$
H_{n_{i}}=\mathbb{Z} / n_{i} \mathbb{Z} \times \mathscr{X}\left(\mathbb{Z} / n_{i} \mathbb{Z}\right) \cong\left(\mathbb{Z} / n_{i} \mathbb{Z}\right)^{2}, \quad e_{d_{i}}\left((x, \chi),\left(x^{\prime}, \chi^{\prime}\right)\right)=\chi^{\prime}(x)^{d_{i}} \chi\left(x^{\prime}\right)^{-d_{i}},
$$

where the $n_{i}$ and $d_{j}$ are integers satisfying $n_{i+1} \mid n_{i}, 0 \leq d_{i}<n_{i}$, and $\left(d_{i}, n_{i}\right)=1$ for all $i$. Moreover, $n_{1}, \ldots, n_{r}$ are uniquely determined by $H$. Since $H$ is a subgroup of $\widehat{X}_{n} \cong(\mathbb{Z} / n \mathbb{Z})^{2 g}$, where $g:=\operatorname{dim}(X)$, we see that $r \leq g$; conversely, any product of $r$ cyclic groups of order prime to $p$ can be embedded into $\widehat{X}_{n}$ provided that $r \leq g$.

It follows that every homogeneous irreducible bundle admits a decomposition into a product

$$
P=P_{1} \cdots P_{r},
$$

where each $P_{i}$ corresponds to $\left(H_{n_{i}}, e_{d_{i}}\right)$. Moreover, the $P_{i}$ are exactly the irreducible homogeneous bundles associated with a product of two copies of a cyclic group; we may call these bundles cyclic.

Equivalently, the associated Azumaya algebra satisfies

$$
\mathscr{A}=\mathscr{A}_{1} \otimes \cdots \otimes \mathscr{A}_{r}
$$

where $\mathscr{A}_{i}$ corresponds to $\left(H_{n_{i}}, e_{d_{i}}\right)$. Moreover, the $\mathscr{O}_{X}$-algebra $\mathscr{A}_{i}$ is generated by two invertible sheaves $\mathscr{L}$ and $\mathcal{M}$ (associated with the natural generators of $\left.\left(\mathbb{Z} / n_{i} \mathbb{Z}\right)^{2}\right)$, with relations $x^{n_{i}}=\xi, y^{n_{i}}=\eta$, and $x y=\zeta^{d_{i}} y x$ for any local generators $x \in \mathscr{L}$ and $y \in \mathcal{M}$, where $\xi$ (resp. $\eta$ ) denotes a local trivialization of $\mathscr{L}^{\otimes n}$ (resp. $\mathcal{M}^{\otimes n}$ ), and $\zeta$ is a fixed primitive $d_{i}$-th root of unity (this follows by combining the isomorphism of algebras (5) with the description of the $H_{n_{i}}$-algebra $\mathrm{M}_{n_{i}}$ obtained in Lemma 2.6(i)). In particular, $\mathscr{A}_{i}$ yields a cyclic division algebra over $k(X)$.

Example 3.3. Let $X$ be an elliptic curve. Then $X$ is canonically isomorphic to $\widehat{X}$ and the finite subgroups of $X$ admitting a nondegenerate alternating pairing are exactly the $n$-torsion subgroups $X_{n}$. In view of the above remark, it follows that the irreducible homogeneous bundles over $X$ are exactly the cyclic bundles. By Theorem 10 of [Atiyah 1957], they are exactly the projectivizations of the indecomposable vector bundles of coprime rank and degree, that is, of the simple vector bundles. 
Example 3.4. Returning to an arbitrary abelian variety $X$, we recall from [Mumford $1966, \S 1]$ a geometric construction of Heisenberg groups. Let $L$ be a line bundle on $X$, and $K(L)$ the kernel of the polarization homomorphism

$$
\varphi_{L}: X \rightarrow \widehat{X}, \quad x \mapsto T_{x}^{*}(L) \otimes L^{-1} .
$$

Denoting by $\mathscr{G}(L)$ the group scheme of automorphisms of the variety $L$ which commute with the action of $\mathbb{G}_{m}$ by multiplication on fibers, we have a central extension

$$
1 \rightarrow \mathbb{G}_{m} \rightarrow \mathscr{G}(L) \rightarrow K(L) \rightarrow 1
$$

The associated commutator pairing on $K(L)$ is denoted by $e^{L}$.

Also, recall that an effective line bundle $L$ is ample if and only if $\varphi_{L}$ is an isogeny; equivalently, $K(L)$ is finite. Then the theta group $\mathscr{G}(L)$ is nondegenerate, and acts on the space of global sections $H^{0}(X, L)$ via its standard representation. Thus, $K(L)$ acts on the associated projective space

$$
|L|:=\mathbb{P}\left(H^{0}(X, L)\right)
$$

and the natural map

$$
f: X \times^{K(L)}|L| \rightarrow X / K(L) \cong \widehat{X}
$$

is an irreducible homogeneous bundle.

As will be shown in detail in Section 4, this bundle is the projectivization of a natural vector bundle $E$ over $\widehat{X}$. Moreover, if $X$ is an elliptic curve (so that $X \cong \widehat{X}$ ) and $L$ has degree $n$, then $E$ has rank $n$ and degree -1 .

We now obtain several criteria for a homogeneous projective bundle to be irreducible:

Proposition 3.5. The following conditions are equivalent for a homogeneous bundle P:

(i) P is irreducible.

(ii) $P$ admits no proper homogeneous subbundle.

(iii) ad $P$ splits into a direct sum of nonzero algebraically trivial line bundles.

(iv) $H^{0}(X, \operatorname{ad}(P))=0$.

(v) $\operatorname{Aut}_{X}(P)$ is finite.

If $P$ is the projectivization of a (semihomogeneous) vector bundle $E$, then $P$ is irreducible if and only if $E$ is simple. 
Proof. (i) $\Longrightarrow$ (ii) follows from Theorem 2.1(iii).

(i) $\Longrightarrow$ (ii) follows from Proposition 3.1(ii).

(iii) $\Longrightarrow$ (iv) holds since $H^{0}(X, L)=0$ for any nonzero $L \in \widehat{X}$.

(iv) $\Longrightarrow$ (v) follows from the fact that $\operatorname{Lie} \operatorname{Aut}_{X}(P)=H^{0}(X, \operatorname{ad}(P))$.

(v) $\Longrightarrow$ (i) By Proposition 2.9, $P$ is indecomposable and the quotient GL( $U)^{H_{u}} / \mathbb{G}_{m}$ id is finite, where $U$ is the indecomposable representation of $H_{u}$ given by that proposition. But $\operatorname{GL}(U)^{H_{u}} / \mathbb{G}_{m}$ id has positive dimension for any unipotent subgroup scheme $H_{u} \subset \mathrm{GL}(U)$, unless $\operatorname{dim}(U)=1$; in the latter case, $P$ is clearly irreducible.

The final assertion follows from the equivalence of (i) and (iv) in view of the isomorphism

$$
H^{0}(X, \operatorname{ad}(\mathbb{P}(E))) \cong H^{0}(X, \operatorname{End}(E)) / k \text { id } .
$$

Remark 3.6. The indecomposable homogeneous bundles are exactly the products $\mathbb{P}(U) I$, where $U$ is an indecomposable unipotent vector bundle, and $I$ an irreducible homogeneous bundle (as follows from Proposition 2.9).

In particular, the indecomposable homogeneous bundles over an elliptic curve $X$ are exactly the projectivizations $\mathbb{P}(U \otimes E)$, where $U$ is as above, and $E$ is a simple vector bundle (as in Example 3.3).

By a result of [Atiyah 1957], any indecomposable vector bundle over $X$ is isomorphic to $U \otimes E \otimes L$ for $U$ and $E$ as above and $L$ a line bundle. Also, $U$ is uniquely determined by its rank; moreover, $E$ is uniquely determined by its (coprime) rank and degree, up to tensoring with a line bundle of degree 0 .

Next, we obtain a cohomological criterion for a bundle to be homogeneous and irreducible, thereby extending a result of Mukai [1978, Theorem 5.8] about simple semihomogeneous vector bundles:

Proposition 3.7. A bundle $P$ is homogeneous and irreducible if and only if we have $H^{0}(X, \operatorname{ad}(P))=H^{1}(X, \operatorname{ad}(P))=0$; then $H^{i}(X, \operatorname{ad}(P))=0$ for all $i \geq 0$.

Proof. Recall that $H^{i}(X, L)=0$ for all $i \geq 0$ and all nonzero $L \in \widehat{X}$. By Proposition 3.1(ii), the same holds with $L$ replaced with ad $P$, if $P$ is homogeneous and irreducible.

For the converse, observe that $\operatorname{ad}(P)=\pi_{*}\left(T_{Y / X}\right)^{\mathrm{PGL}_{n}}$, where $\pi: Y \rightarrow X$ denotes the $\mathrm{PGL}_{n}$-torsor associated to $P$, and $T_{Y / X}$ the relative tangent bundle. Thus, ad $P$ sits in an exact sequence

$$
0 \rightarrow \operatorname{ad}(P) \rightarrow \pi_{*}\left(T_{Y}\right)^{\mathrm{PGL}_{n}} \rightarrow T_{X} \rightarrow 0
$$

obtained from the standard exact sequence $0 \rightarrow T_{Y / X} \rightarrow T_{Y} \rightarrow \pi^{*}\left(T_{X}\right) \rightarrow 0$ by taking the invariant direct image under $\pi$. If $H^{1}(X, \operatorname{ad}(P))=0$, then the natural 
map

$$
H^{0}\left(Y, T_{Y}\right)^{\mathrm{PGL}_{n}}=H^{0}\left(X, \pi_{*}\left(T_{Y}\right)^{\mathrm{PGL}_{n}}\right) \rightarrow H^{0}\left(X, T_{X}\right)
$$

is surjective. But $H^{0}\left(Y, T_{Y}\right)^{\mathrm{PGL}_{n}} \cong \operatorname{Lie}\left(\operatorname{Aut}^{\mathrm{PGL}_{n}}(Y)\right)$ and $H^{0}\left(X, T_{X}\right) \cong \operatorname{Lie}(\operatorname{Aut}(X))$; moreover, $\operatorname{Aut}^{\mathrm{PGL}_{n}}(Y)=\operatorname{Aut}(P)$ is smooth by Remark 2.5, and $\operatorname{Aut}(X)$ is smooth as well. Hence the homomorphism $\operatorname{Aut}^{\mathrm{PGL}_{n}}(Y) \rightarrow \operatorname{Aut}(X)$ is surjective on neutral components, that is, $Y$ is homogeneous. Thus, $P$ is homogeneous, too. If in addition $H^{0}(X, \operatorname{ad}(P))=0$, then $P$ is irreducible by Proposition 3.5.

Remark 3.8. The above argument shows that a bundle $P$ is homogeneous if it satisfies $H^{1}(X, \operatorname{ad}(P))=0$. This may also be seen as follows: observe that $\operatorname{ad}(P)=$ $f_{*}\left(T_{P / X}\right)$ (as follows, for example, by considering an étale trivialization of $P$ ). Moreover, $R^{i} f_{*}\left(T_{P / X}\right)=0$ for all $i \geq 1$, since $H^{i}\left(\mathbb{P}^{n-1}, T_{\mathbb{P} n-1}\right)=0$ for all such $i$. As a consequence, $H^{1}\left(P, T_{P / X}\right)=0$. Then $f$ is rigid as a morphism with target $X$ in view of [Sernesi 2006, Corollary 3.4.9]. It follows readily that $P$ is homogeneous.

The converse statement does not hold, for example, when $X$ is an elliptic curve in characteristic zero, $U_{n}$ is the indecomposable unipotent vector bundle of rank $n \geq 2$, and $P=\mathbb{P}\left(U_{n}\right)$. Then

$$
\operatorname{ad}(P) \cong\left(U_{n} \otimes U_{n}^{*}\right) / k \text { id } \cong U_{2 n-1} \oplus U_{2 n-3} \oplus \cdots \oplus U_{3},
$$

and hence $H^{0}(X, \operatorname{ad}(P))$ has dimension $n-1$. By the Riemann-Roch theorem, the same holds for $H^{1}(X, \operatorname{ad}(P))$.

Projectivizations of vector bundles. In this subsection, we characterize those homogeneous projective bundles that are projectivizations of (not necessarily homogeneous) vector bundles. We first consider a special class of bundles, defined as follows.

Given a positive integer $m$, not divisible by $p$, we say that a bundle $P$ is trivialized by $m_{X}$ (the multiplication by $m$ in $X$ ) if the pull-back bundle $m_{X}^{*}(P) \rightarrow X$ is trivial.

In fact, every such bundle is homogeneous, as a consequence of the following:

Proposition 3.9. (i) A bundle $P$ is trivialized by $m_{X}$ if and only if $P \cong X \times{ }^{X_{m}} \mathbb{P}^{n-1}$ as bundles over $X \cong X / X_{m}$, for some action of $X_{m}$ on $\mathbb{P}^{n-1}$.

(ii) Any irreducible homogeneous $\mathbb{P}^{n-1}$-bundle is trivialized by $n_{X}$.

Proof. (i) If $P$ is trivialized by $m_{X}$, then we have a cartesian square

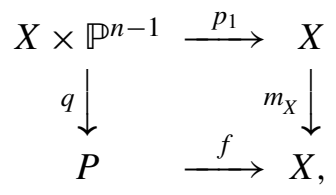


where $p_{1}$ denotes the first projection. Thus, the action of $X_{m}$ by translations on $X$ lifts to an action on $X \times \mathbb{P}^{n-1}$ such that $q$ is invariant. This action is of the form

$$
x \cdot(y, z)=(x+y, \varphi(x, y) \cdot z)
$$

for some morphism $\varphi: X_{m} \times X \rightarrow \operatorname{Aut}\left(\mathbb{P}^{n-1}\right)=\mathrm{PGL}_{n}$. But every morphism from the abelian variety $X$ to the affine variety $\mathrm{PGL}_{n}$ is constant. Thus, $\varphi$ is independent of $y$, that is, $\varphi$ yields an action of $X_{m}$ on $\mathbb{P}^{n-1}$. Moreover, the $X_{m}$-invariant morphism $q$ factors through a morphism of $\mathbb{P}^{n-1}$-bundles $X \times^{X_{m}} \mathbb{P}^{n-1} \rightarrow P$ which is the desired isomorphism.

The converse implication is obvious.

(ii) Write $P=G \times{ }^{H} \mathbb{P}^{n-1}$ as in Theorem 2.1 ; then $H$ is killed by $n$ in view of the structure of nondegenerate theta groups. In other words, the homomorphism $\gamma: G \rightarrow X$ is an isogeny with kernel killed by $n$. Thus, there exists a unique isogeny $\tau: X \rightarrow G$ such that $\gamma \tau=n_{X}$. Then $X_{n}=\tau^{-1}(H)$ and hence $X=X \times^{X_{n}} \mathbb{P}^{n-1}$, where $X_{n}$ acts on $\mathbb{P}^{n-1}$ via the surjective homomorphism $\tau_{\mid X_{n}}: X_{n} \rightarrow H$.

By the above proposition, a bundle $P$ trivialized by $m_{X}$ defines an alternating bilinear map

$$
e_{P, m}: X_{m} \times X_{m} \rightarrow \mu_{m} .
$$

Moreover, the irreducible homogeneous bundles are classified by those maps such that $\left[X_{m}: X_{m}^{\perp}\right]=m^{2}$ (as follows from Proposition 3.5). Also, one easily checks that the assignment $P \mapsto e_{P, m}$ is multiplicative, that is, $e_{P_{1} P_{2}, m}=e_{P_{1}, m} e_{P_{2}, m}$ and $e_{P^{*}, m}=e_{P, m}^{-1}$.

We may now obtain the desired characterization:

Proposition 3.10. Let $P$ be a bundle trivialized by $m_{X}$. Then $P$ is the projectivization of a vector bundle if and only if there exists a line bundle $L$ on $X$ such that $e_{P, m}=\left.e^{L^{\otimes m}}\right|_{X_{m}}$ (this makes sense as $K\left(L^{\otimes m}\right)$ contains $\left.X_{m}\right)$.

Proof. Assume that $P=\mathbb{P}(E)$ for some vector bundle $E$ of rank $n$ on $X$. Since the projective bundle $m_{X}^{*}(\mathbb{P}(E))$ is trivial, we have

$$
m_{X}^{*}(E) \cong M^{\oplus n}
$$

for some line bundle $M$ on $X$. Replacing $E$ with $E \otimes N$, where $N$ is a symmetric line bundle on $X$, leaves $\mathbb{P}(E)$ unchanged and replaces $m_{X}^{*}(E)$ with $m_{X}^{*}(E) \otimes N^{\otimes m^{2}}$, and hence $M$ with $M \otimes N^{\otimes m^{2}}$. Taking for $N$ a large power of an ample symmetric line bundle, we may assume that $M$ is very ample.

The pull-back $m_{X}^{*}(E)$ is equipped with an $X_{m}$-linearization. Equivalently, the action of $X_{m}$ by translations on $X$ lifts to an action on $M^{\oplus n}$ which is linear on fibers. In particular, $T_{x}^{*}\left(M^{\oplus n}\right) \cong M^{\oplus n}$ for any $x \in X_{m}$. This isomorphism is given by an $n \times n$ matrix of maps $T_{x}^{*} M \rightarrow M$; thus, $H^{0}\left(X, T_{x}^{*}\left(M^{-1}\right) \otimes M\right) \neq 0$. Since 
$T_{x}^{*}\left(M^{-1}\right) \otimes M \in \widehat{X}$, it follows that this line bundle is trivial. In other words, $X_{m} \subset$ $K(M)$; this is equivalent to the existence of a line bundle $L$ in $X$ such that $M=L^{\otimes m}$. Moreover, we have a representation of $X_{m}$ in $H^{0}\left(X, M^{\oplus n}\right) \cong H^{0}(X, M) \otimes k^{n}$ that lifts the homomorphism

$$
\phi: X_{m} \rightarrow \operatorname{PGL}\left(H^{0}(X, M)\right) \times \mathrm{PGL}_{n}
$$

given by the $X_{m}$-action on $\mathbb{P}\left(H^{0}(X, M)\right)$ as a subgroup of $K(M)$, and the $X_{m}$-action on $\mathbb{P}^{n-1}$ that defines $P$. It follows that $e^{M} e_{P, m}=1$ on $X_{m}$; equivalently, $e_{P, m}$ is the restriction to $X_{m}$ of $e^{M^{\otimes(-1)}}=e^{L^{\otimes(-m)}}=e^{L^{\otimes m(m-1)}}$ ( since $e^{L^{\otimes m^{2}}}=1$ ).

To show the converse, we reduce by inverting the above arguments to the case that $e^{M} e_{P, m}=1$ on $X_{m}$ for some line bundle $M$ on $X$ such that $X_{m} \subset K(M)$; we may also assume that $M$ is very ample. Then $X_{m}$ acts on $H^{0}\left(X, M^{\oplus n}\right)$ by lifting the homomorphism (18). Moreover, the evaluation morphism

$$
\mathcal{O}_{X} \otimes H^{0}\left(X, M^{\oplus n}\right)=\mathfrak{O}_{X} \otimes H^{0}(X, M) \otimes k^{n} \rightarrow M \otimes k^{n}=M^{\oplus n}
$$

is surjective and its kernel is stable under the induced action of $X_{m}$ (since the analogous morphism $\mathrm{O}_{X} \otimes H^{0}(X, M) \rightarrow M$ is equivariant with respect to the theta group of $X_{m} \subset K(M)$ ). Thus, $X_{m}$ acts on $M^{\oplus n}$ by lifting its action on $X$ via translation. Now $M^{\oplus n}$ descends to a vector bundle on $X / X_{m} \cong X$ with projectivization $P$.

Next, we extend the statement of Proposition 3.10 to all homogeneous bundles $P$. We use the notation of Section 2; in particular, the associated pairing $e_{F}$ introduced in Proposition 2.4. Then $e_{F}$ factors through a nondegenerate pairing on $F / F^{\perp} \cong H / H^{\perp}$ and this group is killed by the homogeneous index $d=d(H)$ defined by (16). Thus, the isogeny $G / H^{\perp} \rightarrow G / H=X$ has its kernel killed by $d$; as in the proof of Proposition 3.9(ii), this yields a canonical surjective homomorphism $X_{d} \rightarrow H / H^{\perp}$ and, in turn, a bilinear alternating pairing $e_{P}$ on $X_{d}$.

Theorem 3.11. With the above notation, $P$ is the projectivization of a vector bundle if and only if $e_{P}=\left.e^{L^{d}}\right|_{X_{d}}$ for some line bundle $L$ on $X$.

Proof. Choose a linear subspace $S \subset \mathbb{P}^{n-1}$ which is $H$-stable, and minimal for this property. Then $S$ yields a homogeneous irreducible $\mathbb{P}^{d-1}$-subbundle of $P$ and the associated pairing on $X_{d}$ is just $e_{P}$. Now the statement is a consequence of Proposition 3.10 together with the following observation.

Lemma 3.12. Let $f: P \rightarrow Z$ be a projective bundle over a nonsingular variety, and $f_{1}: P_{1} \rightarrow Z$ a projective subbundle. Then $P$ is the projectivization of a vector bundle if and only if so is $P_{1}$.

Proof. Clearly, if $P=\mathbb{P}(E)$ for some vector bundle $E$ over $Z$, then $P_{1}=\mathbb{P}\left(E_{1}\right)$ for some subbundle $E_{1} \subset E$. To show the converse, consider the $\mathrm{PGL}_{n}$-torsor 
$\pi: Y \rightarrow Z$ associated with $P$; recall that the subbundle $P_{1}$ yields a reduction of structure group to a $\mathrm{PGL}_{n, n_{1}}$-torsor $\pi_{1}: Y_{1} \rightarrow Z$, where $\mathrm{PGL}_{n, n_{1}} \subset \mathrm{PGL}_{n}$ denotes the stabilizer of $\mathbb{P}^{n_{1}-1} \subset \mathbb{P}^{n-1}$. We have an exact sequence of algebraic groups

$$
1 \longrightarrow G_{n, n_{1}} \longrightarrow \mathrm{PGL}_{n, n_{1}} \stackrel{r}{\longrightarrow} \mathrm{PGL}_{n_{1}} \longrightarrow 1,
$$

where $r$ denotes the restriction to $\mathbb{P}^{n_{1}-1}$ and $G_{n, n_{1}} \cong \mathrm{M}_{n_{1}, n-n_{1}} \ltimes \mathrm{GL}_{n-n_{1}}$, the semidirect product being defined by the natural action of $\mathrm{GL}_{n-n_{1}}$ on the space of matrices $M_{n_{1}, n-n_{1}}$. Also, $\pi_{1}$ factors as

$$
Y_{1} \stackrel{\varphi}{\longrightarrow} Y_{1} / G_{n, n_{1}} \stackrel{\psi}{\longrightarrow} Z,
$$

where $\varphi$ is a $G_{n, n_{1}}$-torsor and $\psi$ is the $\mathrm{PGL}_{n_{1}}$-torsor associated with $P_{1}$. By assumption, $P_{1}=\mathbb{P}\left(E_{1}\right)$ for some vector bundle $E_{1}$; this is equivalent to $\psi$ being locally trivial in view of Proposition 18 of [Serre 2001]. But $\varphi$ is locally trivial as well, since the algebraic group $G_{n, n_{1}}$ is special by Sections 4.3 and 4.4 of that same reference. Thus, $\pi_{1}$ is locally trivial, and hence so is $\pi$. We conclude that $P=\mathbb{P}(E)$ for some vector bundle $E$.

Alternatively, one may use the fact that $P$ is the projectivization of a vector bundle if and only if $f$ has a rational section [loc. cit.], and conclude by applying [Gille and Szamuely 2006, Proposition 5.3.1].

Remark 3.13. We now relate Proposition 3.10 to a description of the Brauer group $\operatorname{Br} X$, due to Berkovich. Recall from [Grothendieck 1968a, §8.4] that $\operatorname{Br} X$ may be viewed as the set of equivalence classes of projective bundles over $X$, where two such bundles $P_{1}$ and $P_{2}$ are equivalent if there exist vector bundles $E_{1}$ and $E_{2}$ such that $\mathbb{P}\left(E_{1}\right) P_{1} \cong \mathbb{P}\left(E_{2}\right) P_{2}$; the group structure stems from the operations of product and duality. By [Berkovich 1972, §3], we have an exact sequence for any positive integer $n$ :

$$
0 \longrightarrow \operatorname{Pic}(X) / n \operatorname{Pic}(X) \stackrel{\varphi}{\longrightarrow} \operatorname{Hom}\left(\Lambda^{2} X_{n}, \mu_{n}\right) \stackrel{\psi}{\longrightarrow} \operatorname{Br}(X)_{n} \longrightarrow 0,
$$

where $\operatorname{Hom}\left(\Lambda^{2} X_{n}, \mu_{n}\right)$ consists of the bilinear alternating pairings $X_{n} \times X_{n} \rightarrow \mu_{n}$ and $\operatorname{Br}(X)_{n} \subset \operatorname{Br}(X)$ denotes the $n$-torsion subgroup; the map $\varphi$ sends the class of $L \in \operatorname{Pic}(X)$ to the pairing $\left.e^{L^{\otimes n}}\right|_{X_{n}}$ and the map $\psi$ sends $e$ to the class of the Azumaya algebra

$$
\mathscr{A}:=\bigoplus_{\alpha \in \widehat{X}_{n}, \sigma \in X_{n}} \mathscr{L}_{\alpha} e_{\sigma},
$$

where $\mathscr{L}_{\alpha}$ denotes the invertible sheaf associated with $\alpha$ and the multiplication is defined by

$$
f_{\alpha} e_{\sigma} \cdot f_{\beta} e_{\tau}=\bar{e}_{n}(\beta, \sigma) a_{\sigma, \tau} f_{\alpha} f_{\beta} e_{\sigma+\tau} .
$$

Here $f_{\alpha}$ (resp. $\left.f_{\beta}\right)$ is a local section of $\mathscr{L}_{\alpha}$ (resp. $\left.\mathscr{L}_{\beta}\right) ; \bar{e}_{n}$ is the canonical pairing between $\widehat{X}_{n}$ and $X_{n}$ and $\left\{a_{\sigma, \tau}\right\} \in Z^{2}\left(X_{n}, \mathbb{G}_{m}\right)$ is a 2-cocycle such that $e(\sigma, \tau)=a_{\sigma, \tau} a_{\tau, \sigma}^{-1}$. 
(The class of $\mathscr{A}$ in the Brauer group does not depend on the choice of the representative $\left\{a_{\sigma, \tau}\right\}$ of $e$ viewed as an element of $H^{2}\left(X_{n}, \mathbb{G}_{m}\right)$.) Thus,

$$
\mathscr{L}:=\bigoplus_{\alpha \in \widehat{X}_{n}} \mathscr{L}_{\alpha} e_{0}
$$

is a maximal étale subalgebra of $\mathscr{A}$ in the sense of [Grothendieck 1968a, définition 5.6]; note that $\mathscr{L} \cong\left(n_{X}\right)_{*} \mathcal{O}_{X}$ as $\mathscr{O}_{X}$-algebras. Moreover, the left $\mathscr{L}$-module $\mathscr{A}$ is free with basis $\left(a_{\sigma}\right)_{\sigma \in X_{n}}$. By [loc. cit., corollaire 5.5], it follows that $n_{X}^{*}(\mathscr{A}) \cong \mathrm{M}_{m}\left(\mathscr{O}_{X}\right)$, where $m:=\#\left(X_{n}\right)=n^{2 g}$. In other words, the projective bundle associated with $\mathscr{A}$ is trivialized by $n_{X}$. In view of Proposition 3.9, it follows that the associated projective bundle is homogeneous.

In fact, any class in $\operatorname{Br}(X)_{n}$ is represented by an irreducible homogeneous bundle. Indeed, given any homogeneous bundle $P$, we may choose an irreducible subbundle $P_{1}$; then the product $P_{1} P_{1}^{*}$ is a subbundle of $P P_{1}^{*}$ and is the projectivization of a vector bundle. By Lemma 3.12, it follows that the class of $P P_{1}^{*}$ in $\operatorname{Br} X$ is trivial; equivalently, $P$ and $P_{1}$ have the same class there.

Recall that the natural map $\operatorname{Br}(X) \rightarrow \operatorname{Br}(k(X))$ is injective (see [Grothendieck $1968 \mathrm{~b}, \S 1])$. Also, as a very special case of a theorem of Merkurjev and Suslin (see [Gille and Szamuely 2006, Theorem 2.5.7]), each class in $\operatorname{Br}(k(X))_{n}$ can be represented by a tensor product of cyclic algebras. So the decomposition of classes in $\operatorname{Br}(X)_{n}$ obtained in Remark 3.2 may be viewed as a global analogue of that result for abelian varieties.

Finally, note that Proposition 3.10 is equivalent to the assertion that the image of $\varphi$ consists of those pairings associated with projectivizations of semihomogeneous vector bundles. In loose terms, the Brauer group is generated by homogeneous bundles and the relations arise from semihomogeneous vector bundles.

\section{Examples}

Let $X$ be an abelian variety, and $\lambda$ an effective class in the Néron-Severi group $N S(X)$ viewed as the group of divisors on $X$ modulo algebraic equivalence. The effective divisors on $X$ with class $\lambda$ are parametrized by a projective scheme $\operatorname{Div}^{\lambda}(X)$. Indeed, the Hilbert polynomial of any such divisor $D$, relative to a fixed ample line bundle on $X$, depends only on $\lambda$; thus, $\operatorname{Div}^{\lambda}(X)$ is a union of connected components of the Hilbert scheme $\operatorname{Hilb}(X)$.

Also, recall that the line bundles on $X$ with class $\lambda$ are parametrized by the Picard variety $\operatorname{Pic}^{\lambda}(X)$. Choosing $L$ in that variety, we have

$$
\operatorname{Pic}^{\lambda}(X)=L \otimes \operatorname{Pic}^{0}(X)=L \otimes \widehat{X}
$$


On $X \times \operatorname{Pic}^{\lambda}(X)$ we have a universal bundle: the Poincaré bundle $\mathscr{P}$, uniquely determined up to the pull-back of a line bundle under the second projection

$$
\pi: X \times \operatorname{Pic}^{\lambda}(X) \rightarrow \operatorname{Pic}^{\lambda}(X) .
$$

The universal family on $\operatorname{Div}^{\lambda}(X)$ yields a morphism

$$
f: \operatorname{Div}^{\lambda}(X) \rightarrow \operatorname{Pic}^{\lambda}(X), \quad D \longmapsto \mathrm{O}_{X}(D) .
$$

Note that $X$ acts on $\operatorname{Div}^{\lambda}(X)$ and on $\operatorname{Pic}^{\lambda}(X)$ via its action on itself by translations; moreover, $f$ is equivariant. Also, the isotropy subgroup scheme in $X$ of any point of $\operatorname{Pic}^{\lambda}(X)$ is the group scheme $K(L)$ that occurred in Example 3.4.

If $\lambda$ is ample, then $\operatorname{Pic}^{\lambda}(X)$ is the $X$-orbit $X \cdot L \cong X / K(L)$. Thus, $f$ is a homogeneous fiber bundle over $X / K(L)$; the latter abelian variety is isomorphic to $\widehat{X}$ via the polarization homomorphism (17).

Proposition 4.1. Let $\lambda \in \operatorname{NS}(X)$ be an ample class, and $L \in \operatorname{Pic}^{\lambda}(X)$.

(i) We have an isomorphism

$$
\operatorname{Div}^{\lambda}(X) \cong X \times \times^{K(L)}|L|
$$

of homogeneous bundles over $X / K(L)$. In particular, $\operatorname{Div}^{\lambda}(X)$ is a homogeneous projective bundle over $\widehat{X}$.

(ii) The sheaf $\mathscr{E}:=\pi_{*}(\mathscr{P})$ is locally free, and the morphism (19) is the projectivization of the corresponding vector bundle.

(iii) The group scheme $\operatorname{Aut}\left(\operatorname{Div}^{\lambda}(X)\right)$ is the semidirect product of $X$ (acting by translations) with the subgroup of $\operatorname{Aut}_{\mathrm{gp}}(X)$ that preserves $K(L)$ and $e^{L}$.

Proof. (i) Clearly, the set-theoretic fiber of $f$ at $L$ is the projective space $|L|$, and its dimension $h^{0}(L)-1=\chi(L)-1$ is independent of $L \in \mathrm{Pic}^{\lambda}(X)$. As a consequence, the scheme $\operatorname{Div}^{\lambda}(X)$ is irreducible of dimension $\operatorname{dim}(X)+h^{0}(L)-1$.

To complete the proof, it suffices to show that the differential of $f$ at any $D \in|L|$ is surjective with kernel of dimension $h^{0}(L)-1$. Identifying $\operatorname{Div}^{\lambda}(X)$ with a union of components of $\operatorname{Hilb}(X)$, and $\operatorname{Pic}^{\lambda}(X)$ with $\widehat{X}$, the differential

$$
T_{D} f: T_{D} \operatorname{Div}^{\lambda}(X) \rightarrow T_{L} \operatorname{Pic}^{\lambda}(X)
$$

is identified with the boundary map $\partial: H^{0}\left(D, L_{\mid D}\right) \rightarrow H^{1}\left(X, O_{X}\right)$ of the long exact sequence of cohomology associated with the short exact sequence

$$
0 \rightarrow \mathrm{O}_{X} \rightarrow L \rightarrow L_{\mid D} \rightarrow 0
$$

(see [Sernesi 2006, Proposition 3.3.6]). Since $H^{1}(X, L)=0$, this long exact sequence begins with

$$
0 \longrightarrow k \longrightarrow H^{0}(X, L) \longrightarrow H^{0}\left(D, L_{\mid D}\right) \stackrel{\partial}{\longrightarrow} H^{1}\left(X, O_{X}\right) \longrightarrow 0
$$


which yields the desired assertion.

(ii) The vanishing of $H^{1}(X, L)$ also implies that $\mathscr{E}$ is locally free and satisfies $\mathscr{E}(L) \cong H^{0}(X, L)$. Thus, it suffices to check that the associated projective bundle $\mathbb{P}(\mathscr{E})$ is homogeneous. But for any $x \in X$, there exists an invertible sheaf $L_{x}$ on $\operatorname{Pic}^{\lambda}(X)$ such that

$$
\left(T_{x}, T_{x}\right)^{*}(\mathscr{P}) \cong \mathscr{P} \otimes \pi^{*} L_{x}
$$

in view of the universal property of the Poincaré bundle $\mathscr{P}$. Since $\pi_{*}\left(T_{x}, T_{x}\right)^{*}(\mathscr{P}) \cong$ $T_{x}^{*}\left(\pi_{*}(\mathscr{P})\right)=T_{x}^{*}(\mathscr{E})$, this yields an isomorphism

$$
T_{x}^{*}(\mathscr{E}) \cong \mathscr{E} \otimes L_{x} .
$$

In other words, $\mathscr{E}$ is semihomogeneous.

(iii) This is checked by arguing as in the proof of Proposition 3.1(iii).

The case of an arbitrary effective class $\lambda$ reduces to the ample case in view of the following:

Proposition 4.2. Let $\lambda \in \mathrm{NS}(X)$ be an effective class, $L \in \operatorname{Pic}^{\lambda}(X)$, and $q: X \rightarrow \bar{X}$ the quotient map by the reduced neutral component $K(L)_{\mathrm{red}}^{0} \subset K(L)$. Then $\lambda=$ $q^{*}(\bar{\lambda})$ for a unique ample class $\bar{\lambda} \in \mathrm{NS}(\bar{X})$, and $f: \operatorname{Div}^{\lambda}(X) \rightarrow \operatorname{Pic}^{\lambda}(X)$ may be identified with $\bar{f}: \operatorname{Div}^{\bar{\lambda}}(\bar{X}) \rightarrow \operatorname{Pic}^{\bar{\lambda}}(\bar{X})$.

Proof. We claim that any $D \in \operatorname{Div}^{\lambda}(X)$ equals $q^{*}(\bar{D})$ for some ample effective divisor $\bar{D}$ on $\bar{X}$.

To see this, recall that $n D$ is base-point-free for any $n \geq 2$; this yields morphisms

$$
\gamma_{n}: X \rightarrow \mathbb{P}\left(H^{0}\left(X, L^{\otimes n}\right)^{*}\right) \quad(n \geq 2),
$$

which are equivariant for the action of $K(L)$. The abelian variety $K(L)_{\text {red }}^{0}$ acts trivially on each projective space $\mathbb{P}\left(H^{0}\left(X, L^{\otimes n}\right)^{*}\right)$; thus, each $\gamma_{n}$ is invariant under $K(L)_{\text {red }}^{0}$. In the Stein factorization of $\gamma_{n}$ as

$$
X \stackrel{\varphi_{n}}{\longrightarrow} Y_{n} \stackrel{\psi_{n}}{\longrightarrow} \mathbb{P}\left(H^{0}\left(X, L^{\otimes n}\right)^{*}\right),
$$

where $\left(\varphi_{n}\right)_{*}\left(\mathcal{O}_{X}\right)=\mathcal{O}_{Y_{n}}$ and $\psi_{n}$ is finite, the morphism $\varphi_{n}$ is the natural map

$$
\varphi: X \rightarrow \operatorname{Proj} \bigoplus_{m=0}^{\infty} H^{0}\left(X, L^{\otimes m}\right)=: Y .
$$

In particular, $\varphi_{n}$ is independent of $n$ and invariant under $K(L)_{\mathrm{red}}^{0}$. Moreover, since $n D$ is the pull-back of a hyperplane under $\gamma_{n}$ for any $n \geq 2$, we see that $D=3 D-2 D=\varphi^{*}(E)$ for some Cartier divisor $E$ on $Y$. Then $E$ is effective 
and $H^{0}\left(X, L^{\otimes n}\right) \cong H^{0}\left(Y, M^{\otimes n}\right)$ for all $n$, where $M:=\mathscr{O}_{Y}(E)$; it follows that $E$ is ample. Consider the factorization

$$
\bar{\varphi}: \bar{X}:=X / K(L)_{\mathrm{red}}^{0} \rightarrow Y,
$$

the effective divisor $\bar{D}:=\bar{\varphi}^{*}(E)$, and the associated invertible sheaf $\bar{L}=\bar{\varphi}^{*}(M)$. Then $L=q^{*}(\bar{L})$. Thus, the group scheme $K(\bar{L})=K(L) / K(L)_{\text {red }}^{0}$ is finite and $\bar{L}$ has nonzero global sections; hence $\bar{L}$ is ample. Thus, $\bar{\varphi}$ is finite. But $\bar{\varphi}_{*}\left(\mathcal{O}_{\bar{X}}\right)=\mathrm{O}_{Y}$; it follows that $\bar{\varphi}$ is an isomorphism, and this identifies $\varphi$ with $q$. This proves the claim.

As a consequence, $\lambda=q^{*}(\bar{\lambda})$ for a unique ample class $\bar{\lambda}$. We now show that the morphism

$$
q^{*}: \operatorname{Div}^{\bar{\lambda}}(\bar{X}) \rightarrow \operatorname{Div}^{\lambda}(X)
$$

is an isomorphism. By the first step, $q^{*}$ is bijective. In view of Proposition 4.1, it follows that the scheme $\operatorname{Div}^{\lambda}(X)$ is irreducible of dimension $\operatorname{dim}(\bar{X})+h^{0}(\bar{X}, \bar{L})-1$. On the other hand, the Zariski tangent space of $\operatorname{Div}^{\lambda}(X)$ at $D$ equals

$$
H^{0}\left(D, L_{\mid D}\right) \cong H^{0}\left(\bar{D}, \bar{L}_{\mid \bar{D}}\right)=T_{\bar{D}} \operatorname{Div}^{\bar{\lambda}}(\bar{X}) .
$$

Thus, $q^{*}$ is étale and hence is an isomorphism.

In the above construction, one may replace the abelian variety $X$ with any smooth projective variety; for example, a curve $C$. Then an effective class in $\operatorname{NS}(C) \cong \mathbb{Z}$ is just a nonnegative integer $d$. Moreover, $\operatorname{Div}^{d}(C)$ is the symmetric product $C^{(d)}$, a smooth projective variety of dimension $d$ equipped with a morphism

$$
f=f_{d}: C^{(d)} \rightarrow \operatorname{Pic}^{d}(C) .
$$

Choosing a point of $C$, we may identify $\operatorname{Pic}^{d}(C)$ with the Jacobian variety $J=J(C)$.

If $d>2 g-2$, where $g$ denotes of course the genus of $C$, then $f$ is the projectivization of a vector bundle $E=E_{d}$ on $\operatorname{Pic}^{d}(C)$, the direct image of the Poincaré bundle on $C \times \operatorname{Pic}^{d}(C)$ under the second projection. Moreover, $E$ has rank $n:=d-g+1$.

Proposition 4.3. With the above notation, the projective bundle (20) is homogeneous if and only if $g \leq 1$.

Proof. Assume that (20) is homogeneous. Then $E$ is semihomogeneous; in view of [Mukai 1978, Lemma 6.11], we then have an isomorphism of vector bundles on $J$,

$$
n_{J}^{*}(E) \cong \operatorname{det}(E)^{\otimes n} \otimes F,
$$

for some homogeneous vector bundle $F$. Moreover, the Chern classes of $F$ are algebraically trivial by [Mukai 1978, Theorem 4.17]. Thus, the total Chern class of $E$ satisfies

$$
n_{J}^{*}(c(E))=\left(1+n c_{1}(E)\right)^{n}
$$


in the cycle ring of $J$ modulo algebraic equivalence. Since $n_{J}^{*}\left(c_{1}(E)\right)=n^{2} c_{1}(E)$ in that ring, this yields

$$
c(E)=\left(1+\frac{c_{1}(E)}{n}\right)^{n} .
$$

We now recall a formula for $c(E)$ due to [Mattuck 1961, Theorem 3]. Denoting by $W_{i}$ the image of $p_{i}$ for $0 \leq i \leq g$, we have

$$
c(E)=\sum_{i=0}^{g}(-1)^{i}\left[W_{g-i}^{-}\right],
$$

where $W_{j}^{-}$denotes the image of $W_{j}$ under the involution $(-1)_{J}$ and the equality holds again modulo algebraic equivalence. In particular,

$$
c_{1}(E)=-\left[W_{g-1}^{-}\right]=-\theta,
$$

where $\theta$ denotes the Chern class of the theta divisor, and

$$
c_{g}(E)=(-1)^{g} e,
$$

where $e$ denotes the class of a point. In view of (21), this yields

$$
e=\left(\begin{array}{l}
n \\
g
\end{array}\right) \frac{\theta^{g}}{n^{g}} .
$$

Since $\theta^{g}=g$ ! e, we obtain $n^{g}=n(n-1) \cdots(n-g+1)$ and hence $g \leq 1$.

Conversely, if $g=0$ then $C^{(d)}=\mathbb{P}^{d}$ and there is nothing to prove; if $g=1$ then the assertion follows from Proposition 4.1.

Remark 4.4. By [Ein and Lazarsfeld 1992], the vector bundle $E$ is stable with respect to the principal polarization of $J$. In particular, $E$ is simple, that is, $\operatorname{Aut}_{J}(P)$ is finite. This yields examples of simple vector bundles on abelian varieties which are not semihomogeneous (see [Oda 1971] for the first construction of bundles satisfying these properties).

\section{Homogeneous self-dual projective bundles}

Generalities on self-dual bundles. Throughout this subsection, we assume that $p \neq 2$; we consider projective bundles over a fixed variety $X$. Let $f: P \rightarrow X$ be a $\mathbb{P}^{n-1}$-bundle, and $f^{*}: P^{*} \rightarrow X$ the dual bundle. By contravariance, any isomorphism of bundles

$$
\varphi: P \rightarrow P^{*}
$$

defines a dual isomorphism $\varphi^{*}: P=P^{* *} \rightarrow P^{*}$. We say that (22) is self-dual if $\varphi^{*}=\varphi$. 
For later use, we now present some general results on self-dual bundles; we omit their (easy) proofs, which can be found in the arXiv version of this article [Brion 2012b].

Proposition 5.1. Given a $\mathbb{P}^{n-1}$-bundle $P$, there is a bijective correspondence between the self-dual morphisms (22) and the reductions of structure group of the associated $\mathrm{PGL}_{n}$-torsor $\pi: Y \rightarrow X$ to a $\mathrm{PO}_{n, \varepsilon}$-torsor $\psi: Z \rightarrow X$, where $\varepsilon= \pm 1$ and $\mathrm{PO}_{n, \varepsilon} \subset \mathrm{PGL}_{n}$ denotes the projective orthogonal (resp. symplectic) group if $\varepsilon=+1($ resp. -1$)$.

We say that the self-dual morphism (22) is symmetric (resp. alternating) if $\varepsilon=1$ (resp. $=-1$ ). Denote by $\mathrm{GO}_{n, \varepsilon}$ the preimage of $\mathrm{PO}_{n, \varepsilon}$ in $\mathrm{GL}_{n}$. Then $\mathrm{GO}_{n, \varepsilon}$ is the stabilizer of a unique line in the space of bilinear forms on $k^{n}$. Moreover, any such semiinvariant form $B$ is nondegenerate, and it is symmetric (resp. alternating) if $\varphi$ has the same property.

The group $\mathrm{GO}_{n, \varepsilon}$ is connected and reductive for any $n$; hence so is $\mathrm{PO}_{n, \varepsilon}$. If $n$ is odd, then we must have $\varepsilon=+1$, and $\mathrm{PO}_{n, \varepsilon}=\mathrm{SO}_{n}$; if $n$ is even, then $\mathrm{PO}_{n,+1}=\mathrm{PSO}_{n}$ and $\mathrm{PO}_{n,-1}=\mathrm{PSp}_{n}$. As a consequence, $\mathrm{PO}_{n, \varepsilon}$ is semisimple of adjoint type unless $n=2$ and $\varepsilon=1$; then $\mathrm{PO}_{2,+1}=\mathbb{G}_{m}$.

Together with the results of Grothendieck recalled on pages 2477-2478 and 2496-2497, Proposition 5.1 yields one-to-one correspondences between self-dual $\mathbb{P}^{n-1}$-bundles (that is, bundles equipped with a self-dual morphism), $\mathrm{PO}_{n, \varepsilon}$-torsors, and Azumaya algebras $\mathscr{A}$ of rank $n^{2}$ equipped with an involution (as in [Parimala and Srinivas 1992]); these correspondences preserve morphisms. The $\mathrm{PO}_{n, \varepsilon}$-torsor $Z \rightarrow X$ corresponds to the associated bundle $P=Z \times{ }^{\mathrm{PO}_{n, \varepsilon}} \mathbb{P}^{n-1} \rightarrow X$ equipped with the isomorphism to $P^{*}$ arising from the $\mathrm{PO}_{n, \varepsilon}$-equivariant isomorphism $\mathbb{P}^{n-1} \cong$ $\left(\mathbb{P}^{n-1}\right)^{*}$ given by $B$. The associated Azumaya algebra is the sheaf of local sections of the matrix bundle $Z \times{ }^{\mathrm{PO}_{n, \varepsilon}} \mathrm{M}_{n}$ equipped with the involution arising from the isomorphism $\mathrm{M}_{n} \rightarrow\left(\mathrm{M}_{n}\right)^{\text {op }}$ defined by the adjoint with respect to the pairing $B$.

Like for $\mathbb{P}^{n-1}$-bundles, we may define the product of the self-dual bundles $\left(P_{i}, \varphi_{i}\right)(i=1,2)$ in terms of the associated $\mathrm{PO}_{n_{i}, \varepsilon_{i}}$-torsors $Z_{i} \rightarrow X$. Specifically, the product $\left(P_{1} P_{2}, \varphi_{1} \varphi_{2}\right)$ corresponds to the $\mathrm{PO}_{n_{1} n_{2}, \varepsilon_{1} \varepsilon_{2}}$-torsor obtained from the $\mathrm{PO}_{n_{1}, \varepsilon_{1}} \times \mathrm{PO}_{n_{2}, \varepsilon_{2}}$-torsor $Z_{1} \times{ }_{X} Z_{2} \rightarrow X$ by the extension of structure groups

$$
\mathrm{PO}_{n_{1}, \varepsilon_{1}} \times \mathrm{PO}_{n_{2}, \varepsilon_{2}}=\mathrm{PO}_{\varepsilon_{1}}\left(k^{n_{1}}\right) \times \mathrm{PO}_{\varepsilon_{2}}\left(k^{n_{2}}\right) \stackrel{\rho}{\rightarrow} \mathrm{PO}_{\varepsilon_{1} \varepsilon_{2}}\left(k^{n_{1}} \otimes k^{n_{2}}\right)=\mathrm{PO}_{n_{1} n_{2}, \varepsilon_{1} \varepsilon_{2}},
$$

where $\rho$ stems from the natural map $\mathrm{GO}_{\varepsilon_{1}}\left(k^{n_{1}}\right) \times \mathrm{GO}_{\varepsilon_{2}}\left(k^{n_{2}}\right) \rightarrow \mathrm{GO}_{\varepsilon_{1} \varepsilon_{2}}\left(k^{n_{1}} \otimes k^{n_{2}}\right)$. This product also corresponds to the tensor product of algebras with involutions, as considered in [Parimala and Srinivas 1992].

Next, we introduce a notion of decomposition of self-dual bundles; for this, we need some observations on duality for subbundles. Any $\mathbb{P}^{n_{1}-1}$-subbundle $P_{1}$ of a bundle $P$ defines a $\mathbb{P}^{n-n_{1}-1}$-subbundle of $P^{*}$, as follows: $P_{1}$ corresponds 
to a $\mathrm{PGL}_{n}$-equivariant morphism $\gamma$ from $Y$ to the Grassmannian $\mathrm{PGL}_{n} / \mathrm{PGL}_{n, n_{1}}$ and hence to an equivariant morphism $\gamma^{*}$ from $Y^{*}$ to the dual Grassmannian, $\mathrm{PGL}_{n} / \mathrm{PGL}_{n, n-n_{1}}$. The latter morphism yields the desired subbundle $P_{1}^{\perp}$. One checks that $P_{1}^{\perp \perp}=P_{1}$ under the identification of $P$ with $P^{* *}$. Moreover, every decomposition $\left(P_{1}, P_{2}\right)$ of $P$ yields a decomposition $\left(P_{2}^{\perp}, P_{1}^{\perp}\right)$ of $P^{*}$, of the same type. We may now define a decomposition of a self-dual bundle $(P, \varphi)$ as a decomposition $\left(P_{1}, P_{2}\right)$ of the bundle $P$, such that $\varphi\left(P_{1}\right)=P_{2}^{\perp}$; then also $\varphi\left(P_{2}\right)=P_{1}^{\perp}$ by self-duality.

Proposition 5.2. Under the correspondence of Proposition 5.1, the decompositions of type $\left(n_{1}, n_{2}\right)$ of $(P, \varphi)$ correspond bijectively to the reductions of structure group of the $\mathrm{PO}_{n, \varepsilon}$-torsor $\mathrm{Z}$ to a $\mathrm{P}\left(\mathrm{O}_{n_{1}, \varepsilon} \times \mathrm{O}_{n_{2}, \varepsilon}\right)$-torsor.

Moreover, each subbundle $P_{i}$ in a decomposition of $(P, \varphi)$ uniquely determines the other one and comes with a self-dual isomorphism $\varphi_{i}: P_{i} \rightarrow P_{i}^{*}$ of the same sign as $\varphi$.

The subbundles $P_{i}$ occurring in a decomposition of $(P, \varphi)$ are characterized by the property that $\varphi\left(P_{i}\right)$ and $P_{i}^{\perp}$ are disjoint; we then say that $P_{i}$ is nondegenerate. A self-dual bundle will be called indecomposable if it admits no proper decomposition; equivalently, any proper subbundle is degenerate.

Remarks 5.3. (1) We also have the notion of L-indecomposability from [Balaji et al. 2005], namely, a self-dual bundle is $L$-indecomposable if the associated $\mathrm{PO}_{n, \varepsilon}$-torsor admits no reduction of structure group to a proper Levi subgroup. The maximal Levi subgroups of $\mathrm{PO}_{n, \varepsilon}$ are exactly the subgroups $\mathrm{P}\left(\mathrm{O}_{n_{1}, \varepsilon} \times \mathrm{GL}_{n_{2}}\right)$, where $n_{1} \geq 0, n_{2} \geq 1, n_{1}+2 n_{2}=n$, and $\mathrm{GL}_{n_{2}} \subset \mathrm{O}_{2 n_{2}, \varepsilon}$ is the subgroup that stabilizes a decomposition $k^{2 n_{2}}=V_{1} \oplus V_{2}$ with $V_{1}$ and $V_{2}$ totally isotropic subspaces of dimension $n_{2}$. Thus, a self-dual bundle is $L$-indecomposable if and only if it admits no proper hyperbolic nondegenerate subbundle, where $(P, \varphi)$ is called hyperbolic if the bundle $P$ has a decomposition $\left(P_{1}, P_{2}\right)$ such that $\varphi\left(P_{i}\right)=P_{i}^{\perp}$ for $i=1,2$.

(2) If $P=\mathbb{P}(E)$ for some vector bundle $E$ over $X$, then the symmetric (resp. antisymmetric) morphisms $\varphi: P \rightarrow P^{*}$ correspond bijectively to the symmetric (resp. antisymmetric) nondegenerate bilinear forms $B: E \times E \rightarrow L$, where $L$ is a line bundle and $B$ is viewed up to multiplication by a regular invertible function on $X$.

Also, note that $\mathbb{P}(E)$ is hyperbolic if and only if $E$ admits a splitting

$$
E \cong V \oplus\left(V^{*} \otimes L\right)
$$

for some vector bundle $V$ and some line bundle $L$; then the bilinear form $B$ on $E$ takes values in $L$ and is given by

$$
b(v \oplus(\xi \otimes s), w \oplus(\eta \otimes t))=\langle v, \eta\rangle t+\varepsilon\langle w, \xi\rangle s,
$$


where $\langle-,-\rangle$ denotes the canonical pairing on $V \times V^{*}$.

Structure of homogeneous self-dual bundles. In this subsection, we still assume that $p \neq 2$; we denote by $X$ a fixed abelian variety and by $f: P \rightarrow X$ a $\mathbb{P}^{n-1}$-bundle. We say that a self-dual bundle $(P, \varphi)$ is homogeneous if the corresponding $\mathrm{PO}_{n, \varepsilon^{-}}$ torsor $Z$ of Proposition 5.1 is homogeneous. Then the bundle $P$ is easily seen to be homogeneous.

In view of [Brion 2012a, Theorem 3.1], the structure of homogeneous self-dual bundles is described by a completely analogous statement to Theorem 2.1 , where $\mathrm{PGL}_{n}$ is replaced with $\mathrm{PO}_{n, \varepsilon}$. This reduces the classification of these bundles to that of the commutative subgroup schemes of $\mathrm{PO}_{n, \varepsilon}$ up to conjugacy. Let $H$ be such a subgroup scheme, $\widetilde{H}$ its preimage in $\mathrm{GO}_{n, \varepsilon}$ and $e: H \times H \rightarrow \mathbb{G}_{m}$ the associated commutator pairing. Choose a nondegenerate bilinear form $B$ on $k^{n}=: V$ which is an eigenvector of $\mathrm{GO}_{n, \varepsilon}$; such a form is unique up to scalar. We say that the pair $(\widetilde{H}, B)$ is a self-dual theta group, and $(V, B)$ a self-dual representation. Note that $\widetilde{H}$ is equipped with a character

$$
\beta: \widetilde{H} \rightarrow \mathbb{G}_{m}
$$

such that

$$
(\tilde{x} \cdot B)\left(v_{1}, v_{2}\right)=B\left(\tilde{x}^{-1} v_{1}, \tilde{x}^{-1} v_{2}\right)=\beta(\tilde{x}) B\left(v_{1}, v_{2}\right),
$$

for all $\tilde{x} \in \widetilde{H}$ and $v_{1}, v_{2} \in V$. In particular, $\beta(t)=t^{-2}$ for all $t \in \mathbb{G}_{m}$; we say that $\beta$ has $\mathbb{G}_{m}$-weight -2 . The existence of such a character imposes a strong restriction on the quotient $H / H^{\perp}=F / F^{\perp}$ (where $F$ denotes the group of components of $H_{s}$ and the orthogonals are relative to the pairing $e$ ):

Lemma 5.4. With the above notation, $H / H^{\perp}$ is a 2-elementary finite group; in particular, the homogeneous index of $P$ is a power of 2. Moreover, e factors through a nondegenerate alternating morphism

$$
\text { se }: H / H^{\perp} \times H / H^{\perp} \rightarrow \mu_{2} \text {. }
$$

Proof. Since $\beta(e(x, y))=\chi\left(\tilde{x} \tilde{y} \tilde{x}^{-1} \tilde{y}^{-1}\right)=1$ for all $x, y \in H$ with lifts $\tilde{x}, \tilde{y} \in \tilde{H}$, we see that $e(2 x, y)=e(x, y)^{2}=1$. Thus, $H^{\perp}$ contains $2 H$ (the image of the multiplication by 2 in the commutative group scheme $H$ ), that is, $F$ is killed by 2. Since $p \neq 2$, this implies the first assertion. For the second, note that $e$ factors through a morphism $\mathrm{H} \times \mathrm{H} \rightarrow \mu_{2}$ and hence through a bilinear alternating morphism (24), which must be nondegenerate by the definition of $H^{\perp}$.

In view of this result, Proposition 2.4, Lemma 2.6, and Proposition 2.7 also hold in this setting (without the assumption that $(n, p)=1$ ), by the same arguments.

We now assume that $e$ is nondegenerate; equivalently, $H^{\perp}$ is trivial. Then we may view $H$ as a finite-dimensional vector space over the field $\mathbb{F}_{2}$ with two elements, and 
se as a symplectic form (with values in $\mathbb{F}_{2}$ ), by identifying $\mathbb{F}_{2}$ to $\mu_{2}$ via $x \mapsto(-1)^{x}$. We denote by $\operatorname{Sp}(H)=\operatorname{Aut}(H, s e)$ the corresponding symplectic group.

Choose a maximal totally isotropic subspace $K \subset H$. Then $H \cong K \oplus K^{*}$ and this identifies $s e$ with the standard symplectic form $\omega$ defined by

$$
\omega\left((x, \xi),\left(x^{\prime}, \xi^{\prime}\right)\right)=\left\langle x, \xi^{\prime}\right\rangle+\left\langle x^{\prime}, \xi\right\rangle,
$$

where $\langle-,-\rangle: K \times K^{*} \rightarrow \mathbb{F}_{2}$ denotes the canonical pairing. In particular, \# $(H)=$ $\#(K)^{2}=2^{2 r}$, where $r:=\operatorname{dim}_{\mathbb{F}_{2}}(K)$, and $\operatorname{Sp}(H)=\operatorname{Sp}_{2 r}\left(\mathbb{F}_{2}\right)$; we say that $r$ is the rank of $(H, e)$. Moreover, the dual $K^{*}$ is identified to the character group of $K$, via the map $\xi \longmapsto\left(x \mapsto(-1)^{\langle x, \xi\rangle}\right)$. Recall that $\widetilde{H}$ is isomorphic to the Heisenberg group $\mathscr{H}(K)$, and has a unique irreducible representation of weight 1: the standard representation in $\mathcal{O}(K)$, of dimension $2^{r}$.

We now analyze the representation of $\widetilde{H}$ in the space of bilinear forms on $W$. Since $p \neq 2$, we have a decomposition of representations $W^{*} \otimes W^{*}=S^{2} W^{*} \oplus \Lambda^{2} W^{*}$ into the symmetric and the alternating components. For any $x \in K$, denote by $\epsilon_{x} \in W^{*}$ the evaluation at $x$, that is, $\epsilon_{x}(f)=f(x)$ for any $f \in W$. Then the $\epsilon_{x}$ $(x \in K)$ form a basis of $W^{*}$ and satisfy

$$
(t, x, \xi) \cdot \epsilon_{y}=t^{-1}(-1)^{\langle x+y, \xi\rangle} \epsilon_{x+y} .
$$

Define bilinear forms on $W$ by

$$
B_{x, \xi}:=\sum_{y \in K}(-1)^{\langle y, \xi\rangle} \epsilon_{y} \otimes \epsilon_{x+y} \quad\left(x \in K, \xi \in K^{*}\right) .
$$

Lemma 5.5. With the above notation, each $B_{x, \chi}$ is an eigenvector of $\widetilde{H}$ with weight

$$
\chi_{x, \xi}:(t, y, \eta) \longmapsto t^{-2}(-1)^{\langle x, \eta\rangle+\langle y, \xi\rangle} .
$$

Also, $B_{x, \xi}$ is symmetric (resp. alternating) if and only if $\langle x, \xi\rangle=0($ resp. $=1)$.

Moreover, the $B_{x, \xi}$ form a basis of $W^{*} \otimes W^{*}$.

Proof. The first assertion is easily checked. It implies the second assertion, since the $B_{x, \chi}$ have pairwise distinct weights and their number is $\#(K)^{2}=\operatorname{dim}\left(W^{*} \otimes W^{*}\right)$.

The normalizer $N_{\mathrm{GL}(W)}(\tilde{H})$ acts on $W^{*} \otimes W^{*}$; it stabilizes $S^{2} W^{*}$ and $\Lambda^{2} W^{*}$, and permutes the eigenspaces of $\widetilde{H}$. Thus, $N_{\mathrm{GL}(W)}(\widetilde{H})$ acts on the set of their weights,

$$
\mathscr{X}:=\left\{\chi_{x, \xi} \mid x \in K, \xi \in K^{*}\right\} .
$$

Note that $\mathscr{X}$ is exactly the set of characters of $\widetilde{H}$ with $\mathbb{G}_{m}$-weight -2 . This is an affine space with underlying vector space the character group of $H$, that we identify with $H$ via the pairing se. Also, $N_{\mathrm{GL}(W)}(\widetilde{H})$ acts on $\mathscr{X}$ by affine automorphisms, and the subgroup $\widetilde{H}$ of $N_{\mathrm{GL}(W)}(\widetilde{H})$ acts trivially, since $\widetilde{H}$ acts on itself by conjugation. 
In view of Lemma 2.6, it follows that $N_{\mathrm{GL}(W)}(\widetilde{H})$ acts on $\mathscr{X}$ via its quotient $\operatorname{Sp}(H)$; the linear part of this affine action is the standard action of $\operatorname{Sp}(H)$ on $H$.

Proposition 5.6. The above action of $\operatorname{Sp}(H)$ on $\mathscr{X}$ has two orbits: the symmetric characters $\chi_{x, \xi}$, where $\langle x, \xi\rangle=0$, and the alternating characters. In particular, $S^{2} W^{*}$ and $\Lambda^{2} W^{*}$ are irreducible representations of $N_{\mathrm{GL}(W)}(\widetilde{H})$.

Proof. Consider the general linear group $\mathrm{GL}(K) \cong \mathrm{GL}_{r}\left(\mathbb{F}_{2}\right)$ acting naturally on $\mathcal{O}(K)=W$. Then one readily checks that this action is faithful and normalizes $\widetilde{H}$; also, the resulting homomorphism $\mathrm{GL}(K) \rightarrow N_{\mathrm{GL}(W)}(\widetilde{H})$ lifts the (injective) homomorphism $\mathrm{GL}(K) \rightarrow \mathrm{Sp}(H)$ associated with the natural representation of $\mathrm{GL}(K)$ in $K \oplus K^{*}$. Moreover, the induced action of $\mathrm{GL}(K)$ on $\mathscr{X}$ is given by $\gamma \cdot \chi_{x, \xi}=\chi_{\gamma(x), \gamma(\xi)}$. Since the pairs $(x, \xi)$ such that $\langle x, \xi\rangle=1$ form a unique orbit of $\mathrm{GL}(K)$, we see that $\mathrm{Sp}(H)$ acts transitively on the alternating characters.

On the other hand, the pairs $(x, \xi)$ such that $\langle x, \xi\rangle=0$ decompose into orbits of $\mathrm{GL}(K)$ according to the (non)vanishing of $x$ and $\xi$; this yields four orbits if $m \geq 2$, and three orbits if $m=1$ (then the orbit with $x \neq 0 \neq \xi$ is missing). Note that the unique GL $(K)$-fixed point $\chi_{0,0}$ (a symmetric weight) is not fixed by $\operatorname{Sp}(H)$ : otherwise, the latter group would act on $\mathscr{X}$ via its representation on $H$, and hence would act transitively on $\mathscr{X} \backslash\left\{\chi_{0,0}\right\} \cong H \backslash\{0\}$. But this is impossible, since $\operatorname{Sp}(H)$ preserves the symmetric weights. Also, note that $\mathrm{GL}(K)$ has index 2 in its normalizer $N_{\mathrm{Sp}(H)}(\mathrm{GL}(K))$; moreover, any element of $N_{\mathrm{Sp}(H)}(\mathrm{GL}(K)) \backslash \mathrm{GL}(K)$ fixes $\chi_{0,0}$ and exchanges the GL(K)-orbits $\left\{\chi_{x, 0} \mid x \in K, x \neq 0\right\}$ and $\left\{\chi_{0, \xi} \mid \xi \in K^{*}\right.$, $x \neq 0\}$.

As a consequence, $\operatorname{Sp}(H)$ acts transitively on the symmetric characters if $m=1$. We now show that this property also holds when $m \geq 2$. In view of Lemma 2.6, it suffices to construct automorphisms $u, v \in \operatorname{Aut}^{\mathbb{G}_{m}}(\widetilde{\widetilde{H}})$ such that $u\left(\chi_{x, 0}\right)=\chi_{x, \xi}=$ $v\left(\chi_{0, \xi}\right)$ for some nonzero $x \in K$ and $\xi \in K^{*}$. For this, let $q: K \rightarrow \mathbb{F}_{2}$ be a quadratic form, and $\varphi: K \rightarrow K^{*}$ the associated alternating map, defined by $\langle\varphi(x), y\rangle=q(x+y)+q(x)+q(y)$. Let $u=u_{q}: \widetilde{H} \rightarrow \widetilde{H}$ be the map such that $u(t, x, \xi)=\left(t(-1)^{q(x)}, x, \xi+\varphi(x)\right)$. Then one may check that $u \in \operatorname{Aut}^{\mathbb{G}_{m}}(\widetilde{H})$ and $u\left(\chi_{x, 0}\right)=\chi_{x, \varphi(x)}$. Since we may choose $q$ so that $\varphi(x) \neq 0$, this yields the desired automorphism $u$ (and $v$ by symmetry).

By Lemma 5.5 and Proposition 5.6, there are exactly two isomorphism classes of self-dual nondegenerate theta groups of a prescribed rank, the isomorphism type being just the "sign". We now construct representatives of each class; we first consider the case of rank 1 . Then $H=\mathbb{F}_{2}^{2} \cong(\mathbb{Z} / 2 \mathbb{Z})^{2}$ has a faithful homomorphism to $\mathrm{PGL}_{2}$, unique up to conjugation. Thus, $H$ lifts to two natural subgroups of $\mathrm{GL}_{2}$ : the dihedral group $D \subset \mathrm{O}_{2}$, and the quaternionic group $Q \subset \mathrm{Sp}_{2}=\mathrm{SL}_{2}$. Both groups are finite of order eight; moreover, $\widetilde{H}_{1}:=\mathbb{G}_{m} D\left(\right.$ resp. $\left.\widetilde{H}_{0}:=\mathbb{G}_{m} Q\right)$ is a 
nondegenerate theta group of rank 1 equipped with a symmetric (resp. alternating) semiinvariant bilinear form.

For an arbitrary rank $r$, the central product $\widetilde{H}_{1} \cdots \widetilde{H}_{1}$ of $r$ copies of $\widetilde{H}_{1}$ (the quotient of the product $\widetilde{H}_{1} \times \cdots \times \widetilde{H}_{1}$ by the subtorus $\left.\left\{\left(t_{1}, \ldots, t_{r}\right) \mid t_{1} \cdots t_{r}=1\right\}\right)$ is a self-dual nondegenerate theta group of rank $r$ and sign +1 . Similarly, the central product of $\widetilde{H}_{0}$ with $r-1$ copies of $\widetilde{H}_{1}$ is a self-dual nondegenerate theta group of rank $r$ and sign -1 .

Remark 5.7. The above description of the self-dual nondegenerate theta groups may also be deduced from the structure of extraspecial 2-groups, that is, of those finite groups $G$ such that the center $Z$ has order two, and $G / Z$ is 2-elementary (see [Huppert 1967, Kapitel III, Satz 13.8] or [Gorenstein 1980, Chapter 5, Theorem 5.2]). Namely, by Lemma 5.4, every self-dual nondegenerate theta group yields an extension $1 \rightarrow \mu_{2} \rightarrow G \rightarrow H \rightarrow 1$, where $G$ is extraspecial. Yet the approach followed here is more self-contained.

Returning to an arbitrary self-dual theta group ( $\widetilde{H} \subset \mathrm{GL}(V), B)$, we now investigate the decomposition of $V$ into eigenspaces $V_{\lambda}$ of $Z\left(\widetilde{H}_{s}\right)$. Recall from Proposition 2.7 that $V_{\lambda} \cong U_{\lambda} \otimes W_{\lambda}$ as a representation of $\widetilde{H} \cong H_{u} \times \widetilde{H}_{s}$, where $W_{\lambda}$ is the standard representation of the Heisenberg group $\widetilde{H}_{s} / \operatorname{ker}(\lambda)$. Also, since $B$ has weight $\beta$, we have $B\left(V_{\lambda}, V_{\mu}\right)=\{0\}$ unless $\lambda+\mu=-\beta$. This readily implies the following observations:

Lemma 5.8. (i) As a self-dual representation, $V$ is the direct sum of the pairwise orthogonal subspaces $V_{\lambda}$, where $2 \lambda=-\beta$, and $V_{\lambda} \oplus V_{-\lambda-\beta}$, where $2 \lambda \neq-\beta$.

(ii) If $2 \lambda=-\beta$, then $U_{\lambda}$ (resp. $W_{\lambda}$ ) is a self-dual representation of $H_{u}$ (resp. $\widetilde{H}_{s} / \operatorname{ker}(\lambda)$ ). Moreover, the restriction of $B$ to $V_{\lambda}$ is the tensor product of the corresponding bilinear forms on $U_{\lambda}$, resp. $W_{\lambda}$.

(iii) If $2 \lambda \neq-\beta$, then $V_{-\lambda-\beta} \cong V_{\lambda}^{*}(-\beta)$ as representations of $\widetilde{H}$. Moreover, the restriction of $B$ to $V_{\lambda} \oplus V_{-\lambda-\beta}$ is given by the symmetrization or alternation of the canonical pairing $V_{\lambda} \otimes V_{\lambda}^{*}(-\beta) \rightarrow k(-\beta)$.

As a direct consequence, we obtain the following analogue of the structure of indecomposable homogeneous bundles (Proposition 2.9):

Proposition 5.9. The following assertions are equivalent for a homogeneous selfdual bundle $(P, \varphi)$ :

(i) $(P, \varphi)$ is indecomposable.

(ii) $V$ is indecomposable as a self-dual representation.

(iii) $\widetilde{H}_{s}$ is a Heisenberg group and one of the following cases occurs: 
(I) $V \cong U \otimes W$, where $U$ is an indecomposable self-dual representation of $H_{u}$ and $W$ is the standard irreducible representation of $\widetilde{H}_{s}$. Moreover, $H_{s}$ is 2-elementary.

(II) $V \cong(U \otimes W) \oplus\left(U^{*} \otimes W^{*}\right)(-\beta)$, where $U$ is an indecomposable representation of $H_{u}, W$ is the standard irreducible representation of $\widetilde{H}_{s}$, and $\beta$ is a character of $\widetilde{H}_{S}$ of weight -2 .

Remarks 5.10. (1) In contrast to Proposition 2.9, there exist indecomposable selfdual bundles $(P, \varphi)$ such that $\operatorname{Aut}_{X}^{0}(P, \varphi)$ is not unipotent. Specifically, if $(P, \varphi)$ is hyperbolic (type (II) above), then the action of $\mathbb{G}_{m}$ on $V$ with weight spaces $U \otimes W$ of weight 1 , and $\left(U^{*} \otimes W^{*}\right)(-\beta)$ of weight -1 , yields a one-parameter subgroup of bundle automorphisms of $(P, \varphi)$.

In fact, the condition that $\operatorname{Aut}_{X}^{0}(P, \varphi)$ is unipotent characterizes $L$-indecomposable self-dual bundles. Also, one easily shows that the homogeneous self-dual bundle $(P, \varphi)$ is $L$-indecomposable if and only if the self-dual representation $V$ contains no nontrivial direct summand of type (II).

(2) If $(P, \varphi)$ is irreducible in the sense that it arises from a nondegenerate theta group, then $\operatorname{Aut}_{X}(P)$ is finite by Proposition 3.5; as a consequence, $\operatorname{Aut}_{X}(P, \varphi)$ is finite. But the converse does not hold in general, for example, for homogeneous selfdual $\mathbb{P}^{2}$-bundles associated with the subgroup $H$ of $\mathrm{PO}_{3}$ generated by the images of the diagonal matrices with coefficients \pm 1 (then $H \cong(\mathbb{Z} / 2 \mathbb{Z})^{2}$ and $e=0$ ). Thus, the criteria for irreducibility in Propositions 3.5 and 3.7 do not extend to self-dual bundles. In [Brion et al. 2012, §7.3], an alternative, group-theoretical notion of irreducibility is introduced for homogeneous principal bundles under a semisimple group in characteristic zero, and Propositions 3.5 and 3.7 are generalized to that setting.

\section{Acknowledgements}

This work originated in a series of lectures given at the Chennai Mathematical Institute in January 2011. I thank that institute and the Institute of Mathematical Sciences, Chennai, for their hospitality, and all the attendants of the lectures, especially V. Balaji, P. Samuel and V. Uma, for their interest and stimulating questions. I also thank C. De Concini, P. Gille, F. Knop, and C. Procesi for very helpful discussions.

\section{References}

[Atiyah 1957] M. F. Atiyah, "Vector bundles over an elliptic curve", Proc. London Math. Soc. (3) 7 (1957), 414-452. MR 24 \#A1274 Zbl 0084.17305

[Balaji et al. 2005] V. Balaji, I. Biswas, and D. S. Nagaraj, "Krull-Schmidt reduction for principal bundles”, J. Reine Angew. Math. 578 (2005), 225-234. MR 2006e:14061 Zbl 1084.14041 
[Balaji et al. 2006a] V. Balaji, I. Biswas, and D. S. Nagaraj, “Tannakian Krull-Schmidt reduction”, J. Reine Angew. Math. 590 (2006), 227-230. MR 2006k:14072 Zbl 1096.14025

[Balaji et al. 2006b] V. Balaji, I. Biswas, D. S. Nagaraj, and A. J. Parameswaran, "Krull-Schmidt reduction of principal bundles in arbitrary characteristic", Expo. Math. 24:3 (2006), 281-289. MR 2007f:14046 Zbl 1097.14012

[Berkovich 1972] V. G. Berkovich, "О группе Брауэра абелевых многообразий”, Funkcional. Anal. i Priložen. 6:3 (1972), 10-15. Translated as “The Brauer group of abelian varieties”, Funct. Anal. Appl. 6:3 (1973), 180-184. MR 46 \#7249 Zbl 0264.14011

[Birkenhake and Lange 2004] C. Birkenhake and H. Lange, Complex abelian varieties, 2nd ed., Grundlehren der Mathematischen Wissenschaften 302, Springer, Berlin, 2004. MR 2005c:14001 Zbl 1056.14063

[Brion 2009] M. Brion, “Anti-affine algebraic groups", J. Algebra 321:3 (2009), 934-952. MR 2009j: 14059 Zbl 1166.14029

[Brion 2011] M. Brion, “On automorphism groups of fiber bundles”, Publ. Mat. Urug. 12 (2011), 39-66. MR 3012239

[Brion 2012a] M. Brion, "Homogeneous bundles over abelian varieties", J. Ramanujan Math. Soc. 27:1 (2012), 91-118. MR 2933488 Zbl 1251.14033

[Brion 2012b] M. Brion, "Homogeneous projective bundles over abelian varieties”, preprint, 2012. arXiv $1104.0818 \mathrm{v} 3$

[Brion et al. 2012] M. Brion, P. Samuel, and V. Uma, Lectures on the structure of algebraic groups and geometric applications, Hindustan Book Agency, New Delhi, 2012.

[Demazure and Gabriel 1970] M. Demazure and P. Gabriel, Groupes algébriques, I: Géométrie algébrique, généralités, groupes commutatifs, Masson, Paris, 1970. MR 46 \#1800 Zbl 0203.23401

[Ein and Lazarsfeld 1992] L. Ein and R. Lazarsfeld, "Stability and restrictions of Picard bundles, with an application to the normal bundles of elliptic curves", pp. 149-156 in Complex projective geometry (Trieste, 1989/Bergen, 1989), edited by G. Ellingsrud et al., London Math. Soc. Lecture Note Ser. 179, Cambridge University Press, Cambridge, 1992. MR 94a:14015 Zbl 0768.14012

[Elencwajg and Narasimhan 1983] G. Elencwajg and M. S. Narasimhan, "Projective bundles on a complex torus”, J. Reine Angew. Math. 340 (1983), 1-5. MR 84h:32034 Zbl 0498.32015

[Gille and Szamuely 2006] P. Gille and T. Szamuely, Central simple algebras and Galois cohomology, Cambridge Studies in Advanced Mathematics 101, Cambridge University Press, Cambridge, 2006. MR 2007k:16033 Zbl 1137.12001

[Gorenstein 1980] D. Gorenstein, Finite groups, 2nd ed., Chelsea, New York, 1980. MR 81b:20002 Zbl 0463.20012

[Grothendieck 1968a] A. Grothendieck, "Le groupe de Brauer, I: algèbres d'Azumaya et interprétations diverses", pp. 46-66 in Dix exposés sur la cohomologie des schémas, Advanced Studies in Pure Mathematics 3, North-Holland, Amsterdam, 1968. MR 39 \#5586a Zbl 0193.21503

[Grothendieck 1968b] A. Grothendieck, "Le groupe de Brauer, II: théorie cohomologique”, pp. 67-87 in Dix exposés sur la cohomologie des schémas, Advanced Studies in Pure Mathematics 3, North-Holland, Amsterdam, 1968. MR 39 \#5586b Zbl 0198.25803

[Herpel 2013] S. Herpel, "On the smoothness of centralizers in reductive groups", Trans. Amer. Math. Soc. 365:7 (2013), 3753-3774. MR 3042602

[Huppert 1967] B. Huppert, Endliche Gruppen, I, Grundlehren der Mathematischen Wissenschaften 134, Springer, Berlin, 1967. MR 37 \#302 Zbl 0217.07201 
[Levy et al. 2009] P. Levy, G. McNinch, and D. M. Testerman, "Nilpotent subalgebras of semisimple Lie algebras", C. R. Math. Acad. Sci. Paris 347:9-10 (2009), 477-482. MR 2011b:17022 Zbl 1175.17006

[Mattuck 1961] A. Mattuck, "Symmetric products and Jacobians", Amer. J. Math. 83 (1961), 189-206. MR 26 \#122 Zbl 0225.14020

[Mukai 1978] S. Mukai, "Semi-homogeneous vector bundles on an abelian variety", J. Math. Kyoto Univ. 18:2 (1978), 239-272. MR 58 \#16667 Zbl 0417.14029

[Mumford 1966] D. Mumford, “On the equations defining abelian varieties, I", Invent. Math. 1 (1966), 287-354. MR 34 \#4269 Zbl 0219.14024

[Mumford 1970] D. Mumford, Abelian varieties, Tata Institute of Fundamental Research Studies in Mathematics 5, Oxford University Press, Oxford, 1970. MR 44 \#219 Zbl 0223.14022

[Oda 1971] T. Oda, "Vector bundles on abelian surfaces", Invent. Math. 13 (1971), 247-260. MR 47 \#6701 Zbl 0216.05903

[Oort 1966] F. Oort, Commutative group schemes, Lecture Notes in Mathematics 15, Springer, Berlin, 1966. MR 35 \#4229 Zbl 0216.05603

[Parimala and Srinivas 1992] R. Parimala and V. Srinivas, "Analogues of the Brauer group for algebras with involution”, Duke Math. J. 66:2 (1992), 207-237. MR 93i:16027 Zbl 0780.13002

[Sancho de Salas and Sancho de Salas 2009] C. Sancho de Salas and F. Sancho de Salas, "Principal bundles, quasi-abelian varieties and structure of algebraic groups", J. Algebra 322:8 (2009), 27512772. MR 2010m:14058 Zbl 1191.14055

[Sernesi 2006] E. Sernesi, Deformations of algebraic schemes, Grundlehren der Mathematischen Wissenschaften 334, Springer, Berlin, 2006. MR 2008e:14011 Zbl 1102.14001

[Serre 2001] J. P. Serre, Espaces fibrés algébriques, pp. 107-139, Documents Mathématiques 1, Soc. Math. France, Paris, 2001.

Communicated by Brian Conrad

Received 2012-09-17 Revised 2013-01-31 Accepted 2013-03-12

michel.brion@ujf-grenoble.fr Institut Fourier, Université Grenoble I, CNRS UMR 5582, 100 rue des Maths, BP 74, 38402 St Martin d'Hères, France 


\section{Algebra \& Number Theory}

msp.org/ant

\section{EDITORS}

MANAGING EDITOR

Bjorn Poonen

Massachusetts Institute of Technology

Cambridge, USA

\author{
EDITORIAL BOARD CHAIR \\ David Eisenbud \\ University of California \\ Berkeley, USA
}

\section{BOARD OF EDITORS}

Georgia Benkart

Dave Benson

Richard E. Borcherds

John H. Coates

J-L. Colliot-Thélène

Brian D. Conrad

Hélène Esnault

Hubert Flenner

Edward Frenkel

Andrew Granville

Joseph Gubeladze

Roger Heath-Brown

Ehud Hrushovski

Craig Huneke

Mikhail Kapranov

Yujiro Kawamata

János Kollár

Yuri Manin

Barry Mazur

Philippe Michel
University of Wisconsin, Madison, USA

University of Aberdeen, Scotland

University of California, Berkeley, USA

University of Cambridge, UK

CNRS, Université Paris-Sud, France

University of Michigan, USA

Freie Universität Berlin, Germany

Ruhr-Universität, Germany

University of California, Berkeley, USA

Université de Montréal, Canada

San Francisco State University, USA

Oxford University, UK

Hebrew University, Israel

University of Virginia, USA

Yale University, USA

University of Tokyo, Japan

Princeton University, USA

Northwestern University, USA

Harvard University, USA

École Polytechnique Fédérale de Lausanne
Susan Montgomery

Shigefumi Mori

Raman Parimala

Jonathan Pila

Victor Reiner

Karl Rubin

Peter Sarnak

Joseph H. Silverman

Michael Singer

Vasudevan Srinivas

J. Toby Stafford

Bernd Sturmfels

Richard Taylor

Ravi Vakil

Michel van den Bergh

Marie-France Vignéras

Kei-Ichi Watanabe

Efim Zelmanov

Shou-Wu Zhang
University of Southern California, USA

RIMS, Kyoto University, Japan

Emory University, USA

University of Oxford, UK

University of Minnesota, USA

University of California, Irvine, USA

Princeton University, USA

Brown University, USA

North Carolina State University, USA

Tata Inst. of Fund. Research, India

University of Michigan, USA

University of California, Berkeley, USA

Harvard University, USA

Stanford University, USA

Hasselt University, Belgium

Université Paris VII, France

Nihon University, Japan

University of California, San Diego, USA

Princeton University, USA

PRODUCTION

production@msp.org

Silvio Levy, Scientific Editor

See inside back cover or msp.org/ant for submission instructions.

The subscription price for 2013 is US $\$ 200 /$ year for the electronic version, and $\$ 350 /$ year $(+\$ 40$, if shipping outside the US) for print and electronic. Subscriptions, requests for back issues and changes of subscribers address should be sent to MSP.

Algebra \& Number Theory (ISSN 1944-7833 electronic, 1937-0652 printed) at Mathematical Sciences Publishers, 798 Evans Hall \#3840, c/o University of California, Berkeley, CA 94720-3840 is published continuously online. Periodical rate postage paid at Berkeley, CA 94704, and additional mailing offices.

ANT peer review and production are managed by EditFLOW ${ }^{\circledR}$ from Mathematical Sciences Publishers.

\section{PUBLISHED BY}

- mathematical sciences publishers

nonprofit scientific publishing

http://msp.org/

(C) 2013 Mathematical Sciences Publishers 


\section{Algebra \& Number Theory}

Volume $7 \quad$ No. $10 \quad 2013$

On Kato's local $\epsilon$-isomorphism conjecture for rank-one Iwasawa modules

OTMAR VENJAKOB

Polyhedral adjunction theory

SANDRA Di Rocco, Christian HaAse, Benjamin Nill and ANDREas

PAFFENHOLZ

Genericity and contragredience in the local Langlands correspondence

TASHO KALETHA

Homogeneous projective bundles over abelian varieties

MICHEL BRION

On the second Tate-Shafarevich group of a 1-motive

PETER JOSSEN

Triangulable $\mathcal{O}_{F}$-analytic $\left(\varphi_{q}, \Gamma\right)$-modules of rank 2

LIONEL FoURQUAUX and BINGYONG XIE 